\title{
A HLLC SCHEME FOR NONCONSERVATIVE HYPERBOLIC PROBLEMS. APPLICATION TO TURBIDITY CURRENTS WITH SEDIMENT TRANSPORT
}

\author{
Manuel Jesús Castro Díaz ${ }^{1}$, Enrique Domingo Fernández-Nieto ${ }^{2}$, \\ Tomás Morales de Luna ${ }^{3}$, Gladys Narbona-Reina ${ }^{3}$ and Carlos Parés ${ }^{1}$
}

\begin{abstract}
The goal of this paper is to obtain a well-balanced, stable, fast, and robust HLLC-type approximate Riemann solver for a hyperbolic nonconservative PDE system arising in a turbidity current model. The main difficulties come from the nonconservative nature of the system. A general strategy to derive simple approximate Riemann solvers for nonconservative systems is introduced, which is applied to the turbidity current model to obtain two different HLLC solvers. Some results concerning the nonnegativity preserving property of the corresponding numerical methods are presented. The numerical results provided by the two HLLC solvers are compared between them and also with those obtained with a Roe-type method in a number of $1 \mathrm{~d}$ and $2 \mathrm{~d}$ test problems. This comparison shows that, while the quality of the numerical solutions is comparable, the computational cost of the HLLC solvers is lower, as only some partial information of the eigenstructure of the matrix system is needed.
\end{abstract}

Mathematics Subject Classification. 65N06, 76B15, 76M20, 76N99.

Received June 24, 2011. Revised April 4, 2012.

Published online July 31, 2012.

\section{INTRODUCTION}

The goal of this paper is to obtain a well-balanced, stable, fast, and robust approximate Riemann solver for the hyperbolic PDE system arising in a turbidity current model studied in [23]. The system consists of (a) the depth-averaged mass and momentum equation for the dilute turbidity current which are similar to the shallow water model, (b) a depth-averaged equation for every species of suspended sediment which is an advection equation with a source term modeling erosion and deposition, and (c) one more equation that models the bedload transport which is based on an empirical solid transport discharge formula. If the number of sediment species in the turbidity current is equal to 0 and the deposition and erosion terms are neglected, the classical

Keywords and phrases. Well-balanced, finite volume method, path-conservative, simple Riemann solver, HLLC.

1 Dpto. de Análisis Matemático, Facultad de Ciencias, Universidad de Málga, Campus de Teatinos, s/n, 29071 Málaga, Spain. castor@anamat.cie.uma.es; pares@anamat.cie.uma.es

2 Dpto. Matemática Aplicada I, ETS Arquitectura, Universidad de Sevilla, Avda. Reina Mercedes No. 2 , 41012 Sevilla, Spain. edofer@us.es; gnarbona@us.es

3 Dpto. de Matemáticas, Universidad de Córdoba, Campus de Rabanales, 14071 Córdoba, Spain. tomas.morales@uco.es 
Saint-Venant-Exner models for the bedload transport are recovered (see [11]). And if the bedload transport is also neglected, the standard shallow water model with geometrical source term is recovered.

In [23] a generalized Roe method was used to discretize this PDE system. As it is well known, a Roe method is based on a linear approximate Riemann solver which is complete: the approximate Riemann solution is composed by a number of simple waves that is equal to the number $N$ of unknowns of the system. To implement such a method, the eigenstructure of the Roe linearisation has to be explicitly known or numerically computed at every inter-cell at every time step, what may be expensive from the computational point of view. In this article we look for numerical methods based on incomplete Riemann solvers (i.e. the approximate solutions are composed by less than $N$ simple waves) whose accuracy is comparable with that of a Roe method but whose computational cost is lower, as only some information about the eigenstructure of the intermediate matrices is required.

A classical example of approximate Riemann solver is the HLL one, introduced by Harten, Lax, and van Leer for systems of balance laws in [16]: the approximate Riemann solutions consist of two simple waves linking three constant states. The HLL solver is at the basis of many efficient and robust Godunov-type methods. Nevertheless, for systems of three or more equations, it becomes an incomplete Riemann solver that may be insufficient to capture some relevant physical features of the exact solutions related to the intermediate characteristic fields, such as contact discontinuities, shear waves, etc. The HLLC Riemann solver (where C stands for Contact) proposed by Toro et al. in [31] introduces one or more intermediate waves to the approximate Riemann solutions in order to capture better some of these features.

The strategy followed here will be to derive an HLLC-type solver for the PDE system under study. The main difficulties come from the nonconservative nature of the system: while in the context of systems of conservation laws the Riemann invariants and the Rankine-Hugoniot conditions provide all the necessary information to derive consistent approximate Riemann solvers, in the presence of nonconservative products the jump conditions can be defined in more than a way. To overcome this difficulty, the definition of approximate Riemann solver for nonconservative systems introduced in [28] will be used. This definition, that generalizes the usual one for conservative systems, is based on the theory developed by Dal Maso, LeFloch and Murat in [10] that allows one to set the jump conditions (and thus the concept of weak solution) in terms of a given family of paths. Unfortunately, the speed of propagation of the discontinuities explicitly depends on the chosen family of paths, so that a good choice of paths is crucial to capture the physical meaningful weak solutions. A possible way to compute the correct paths could be based on the vanishing viscosity method: a parabolic regularization is considered with a small viscosity coefficient and the corresponding viscous profiles are then computed. If two states can be linked by an admissible discontinuity, the path connecting them should be the corresponding viscous profiles: see [29] and the references therein for details. An important difference between conservative and nonconservative system is that, in the nonconservative case, the jump conditions obtained from the vanishing viscosity method explicitly depend on the expression of the viscous term. Therefore, the expression of the vanishing viscous terms should be taken from the physics of the problem. Unfortunately, in the PDE system under study it is not clear what is the correct viscous regularization of the system to be considered: while the choice of the viscous term for the momentum equation of the dilute current is known, there is not even a momentum equation for the sediment layer. Due to this difficulty, here we consider the family of straight segments: this is a sensible choice as it is expected to provide third order approximations of the physical jump conditions (in a sense to be specified) and it also makes easier the design of well-balanced methods. Let us also remark that every finite difference type introduced previously for models based on the same approach of the bedload transport is based on an arbitrary choice of the meaning of the noncoservative terms, although this choice may not be explicitly stated.

Another important difficulty due to the nonconservative nature of the system is related to the convergence of the numerical solutions: due to the effects of the numerical viscosity, the formal consistency of the numerical method with a particular definition of the jump conditions does not imply that the discontinuities appearing at the limits of the numerical approximations are in good agreement with these conditions. This difficulty, that also appears when a conservative system is discretized with a nonconservative method (see [17]), has been studied in [5]. Another example of this phenomenon has been shown in [1]. Nevertheless, our experience shows that the 
convergence errors is small in this particular case: this fact will be illustrated in Section 5. In any case, taking into account the simple approach of the bedload transport considered here, the modelization errors are very likely to be bigger than those related to the definition of weak solutions or the convergence errors.

Let us finally remark that, despite the strong simplification hypotheses used in the derivation of the model and the lack of some good mathematical properties of the PDE system obtained (there is neither a momentum equation for the sediment layer nor an entropy pair) the approach of the bedload transport by means of an empirical solid transport discharge formula is widely spread for practical purposes: $[11,15,22,27,33]$. The range of validity of the models based on this approach has to be analysed by comparison between the numerical solutions and the experimental measurements. We emphasize that the goal of this article is eminently practical: our purpose is to introduce a numerical method with good properties of well-balancing, stability, and low computational cost for the turbidity current model considered. Moreover, as Saint-Venant-Exner and shallow water models are particular cases of the studied model, the numerical methods obtained here can be also applied to them. Let us also mention that, in the latter case, the difficulties related to the definition of weak solutions and the convergence difficulties mentioned above do not appear, provided that the depth function is smooth enough: see [26].

The article is organized as follows: in Section 2 we present the general equations of the turbidity current model. Next, in Section 3 we recall the definition of approximate Riemann solver introduced in [28] which is illustrated by deriving a general expression of the HLL solver for nonconservative systems. While in the case of the HLL it is possible to write the approximate Riemann solver in a very general way, the HLLC solvers strongly depends on the structure of the particular PDE system to be solved. In Section 4 we introduce the general strategy to derive them, which is then applied to the turbidity current model to obtain two different HLLC solvers: the essentially three waves HLLC solver (E3W-HLLC) and the four waves HLLC solver (4W-HLLC). The non-negativity-preserving properties of the corresponding numerical schemes as well as their relationship with the Suliciu relaxation method for the shallow water system introduced in [3] and the HLLC solver introduced in [31] will be discussed. Finally, in Section 5 the numerical solutions obtained with the two HLLC solvers are compared between them and also with those obtained with the Roe method introduced in [23] in some 1d and $2 \mathrm{~d}$ numerical tests. A numerical study of the mentioned convergence difficulty is also presented. Finally some conclusions are drawn.

\section{TURBIDITY CURRENT MODEL}

When a river that flows into the sea carries a high concentration of suspended sediment, to the extent that the density of the river is greater than that of the receiving ambient water, the river plunges into the ocean creating what is called a turbidity current or hyperpycnal plume. This plume can travel significant distances until it loses its identity by entraining surrounding ambient water and dropping its sediment load. In [23] a new model for the simulation of turbidity currents was introduced. This model can be described as follows: let us consider $n_{s} \geq 1$ species of sediments with constant density $\rho_{j}$, for $j=1, \ldots, n_{s}$ transported by a river with freshwater of constant density $\rho_{0}$. The river flows in an ambient fluid (in general the sea) of density $\rho_{w}$.

The governing equations for the dilute turbidity current are as follows:

$$
\left\{\begin{array}{l}
\partial_{t} h+\nabla_{x} \cdot(h \vec{u})=\phi_{\eta}+\phi_{b}, \\
\partial_{t}(h \vec{u})+\nabla_{x} \cdot(h \vec{u} \otimes \vec{u})+\nabla_{x}\left(g\left(R_{0}+R_{c}\right) \frac{h^{2}}{2}\right)=-g\left(R_{0}+R_{c}\right) h \nabla_{x}\left(z_{b}-H\right)+\vec{u} \phi_{\eta}+\frac{\vec{u}}{2} \phi_{b}, \\
\partial_{t}\left(h c_{j}\right)+\nabla_{x} \cdot\left(h c_{j} \vec{u}\right)=\phi_{b}^{j}, \quad j=1, \ldots, n_{s}, \\
\partial_{t} z_{b}+\nabla_{x} q_{b}=-\xi \phi_{b},
\end{array}\right.
$$

where $h$ is the thickness of the plume; $\vec{u}$ is the depth-averaged horizontal velocity; $c_{j}, j=1, \ldots, n_{s}$ represents the vertically averaged volume concentration of the $j$ th sediment species; $c=\sum_{i=1}^{n_{s}} c_{j}$, is the total sediment 


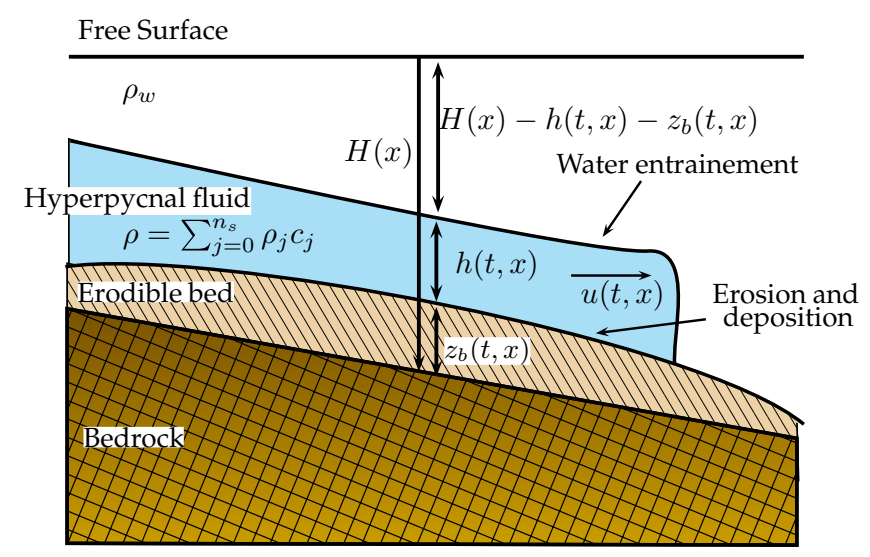

FiguRE 1. Sketch of a turbidity current.

concentration; and

$$
R_{j}=\frac{\rho_{j}-\rho_{0}}{\rho_{0}}, j=1, \ldots, n_{s} ; \quad R_{0}=\frac{\rho_{0}-\rho_{w}}{\rho_{0}} ; \quad \text { and } R_{c}=\sum_{j=1}^{n_{s}} R_{j} c_{j} .
$$

We will denote by $\vec{q}=h \vec{u}$ the discharge.

Remark 2.1. If the ambient fluid is the sea the approximation $\rho_{0} \approx \rho_{w}$ may be considered and thus $R_{0}=0$ and, if the ambient fluid is the air, the approximation $\rho_{w} \approx 0$ may be used and thus $R_{0}=1$.

$H$ is the depth of the non-erodible bottom measured from a reference level (for instance, the undisturbed free surface of the ambient water). $z_{b}$ is the thickness of the sediment layer which may be modified by the fluid by erosion, deposition or bedload transport. $z_{b}-H$ is thus the interface between the bottom and the fluid (see Fig. 1). Finally, $q_{b}=q_{b}\left(h, \vec{u}, c, z_{b}\right)$ represents the solid transport discharge, which is usually given by an empirical formula. Many different expressions of this formula have been proposed in the literature. The formula proposed by Grass in [15] is among the simpler ones:

$$
q_{b}(\vec{u})=\xi A|\vec{u}|^{m-1} \vec{u},
$$

where $A$ is the constant of interaction between the fluid and the sediment layer, $\xi$ is related to the porosity $p \in[0,1)$ by $\xi=\frac{1}{1-p}$, and $m$ is a parameter which is usually set to $m=3$. We refer to [7,23] and the references therein for details about some other possible expressions of $q_{b}$.

Fowler proposed in [13] (see also [24]) a new expression of $q_{b}$ that depends not only on the flow variables, as it is the case in (2.3) but also on the thickness of the sediment layer. This dependence is in agreement with the physics of the problem: if $z_{b}=0$, the solid transport discharge has to be 0 , which is not the case for the Grass model. Therefore, we will consider here the following modified Grass solid formula:

$$
q_{b}=z_{b} \tilde{q}_{b}(h, \vec{u}, c)
$$

with

$$
\tilde{q}_{b}(h, u, c)=\xi \frac{A}{\bar{z}}|\vec{u}|^{m-1} \vec{u},
$$

where, $\bar{z}$ represents the mean value of the thickness of the sediment layer. Nevertheless, the numerical methods obtained in this article are easily adaptable to any of the classical formulae for $\tilde{q_{b}}$. 
The source terms $\phi_{\eta}$ and $\phi_{b}^{j}$ represent respectively the amount of sea water entrained and mixed by turbulence to the plume, and the deposition/erosion flux of the $j$ th sediment species. These source terms are given by different empirical laws which depend on the physical properties of the sediment. In general, water entrainment is described by

$$
\phi_{\eta}=E_{w}|\vec{u}|,
$$

where $E_{w}$ depends on the Richardson number $\mathcal{R} i=\frac{R_{c} g h}{|\vec{u}|^{2}}$.

The sediment flux at the bed of the $j$ th species is determined from the rates of deposition $\left(F_{d}^{j}\right)$ and erosion $\left(F_{e}^{j}\right)$,

$$
\phi_{b}=\sum_{j=1}^{n_{s}} \phi_{b}^{j}, \quad \phi_{b}^{j}=F_{e}^{j}-F_{d}^{j}, \quad F_{d}^{j}=v_{s_{j}} c_{b_{j}}, \quad F_{e}^{j}=v_{s_{j}} p_{j} E_{s_{j}},
$$

where $v_{s_{j}}$ is the settling velocity; $c_{b_{j}}$ is the near bed concentration of sediment which may be described in terms of $c_{j}$; $p_{j}$ is the volumetric concentration of sediment $j$ in the bed; and $E_{s_{j}}$ depends on the velocity $\vec{u}$. Let us remark that there are some discrepancies in the mathematical expression of the source terms $\phi_{\eta}$ and $\phi_{b}^{j}$. The expressions proposed in $[18,20,23,30]$ are among the most used, but some variants may be found in $[2,4,19]$. We refer to these references for further details.

Notice that system (2.1) is invariant under rotation, what allows one to easily derive an approximate Riemann solver for the $2 \mathrm{~d}$ system from a $1 \mathrm{~d}$ one, as it was done in [12]. Therefore, for the sake of simplicity and without loss of generality, we shall only consider the $1 \mathrm{~d}$ case with one sediment species whose concentration is denoted by $c$. We also consider $R_{0}=1$ (see Rem. 2.1). The PDE system reduces then to:

$$
\left\{\begin{array}{l}
\partial_{t} h+\partial_{x}(h u)=\phi_{\eta}+\phi_{b}, \\
\partial_{t}(h u)+\partial_{x}\left(h u^{2}+g(1+R c) \frac{h^{2}}{2}\right)=-g(1+R c) h \partial_{x}\left(z_{b}-H\right)+u \phi_{\eta}+\frac{u}{2} \phi_{b}, \\
\partial_{t}(h c)+\partial_{x}(h u c)=\phi^{b} \\
\partial_{t} z_{b}+\partial_{x} q_{b}=-\xi \phi_{b}
\end{array}\right.
$$

where $R=\left(\rho_{1}-\rho_{0}\right) / \rho_{0}$. We will denote by $q=h u$ the discharge.

This system can be rewritten as follows:

$$
\partial_{t} W+\partial_{x} F(W)+B(W) \partial_{x} W-S(W) \partial_{x} H=G(W)
$$

where $W=\left(h, u h, h c, z_{b}\right)$ and

$$
\begin{aligned}
& F(W)=\left(\begin{array}{c}
h u \\
h u^{2}+\frac{1}{2} g(1+R c) h^{2} \\
h u c \\
q_{b}
\end{array}\right) \\
& B(W)=\left(\begin{array}{lllc}
0 & 0 & 0 & 0 \\
0 & 0 & 0 & g(1+R c) h \\
0 & 0 & 0 & 0 \\
0 & 0 & 0 & 0
\end{array}\right), \quad S(W)=\left(\begin{array}{c}
0 \\
g(1+R c) h \\
0 \\
0
\end{array}\right),
\end{aligned}
$$

and

$$
G(W)=\left(\begin{array}{c}
\phi_{\eta}+\phi_{b} \\
u \phi_{\eta}+\frac{u}{2} \phi_{b} \\
\phi^{b} \\
-\xi \phi_{b}
\end{array}\right)
$$


Finally, system (2.9) can also be rewritten in a more compact form as follows:

$$
\partial_{t}\left(\begin{array}{c}
W \\
H
\end{array}\right)+\left(\begin{array}{c|c}
\mathcal{A}(W) & -S(W) \\
\hline 0 & 0
\end{array}\right) \partial_{x}\left(\begin{array}{c}
W \\
H
\end{array}\right)=\left(\begin{array}{c}
G(W) \\
0
\end{array}\right)
$$

with $\mathcal{A}(W)=J(W)+B(W)$, where $J(W)=\partial_{W} F(W)$ represents the Jacobian matrix of the flux function $F(W)$.

Although (2.8) is not strictly hyperbolic, in [23] it was shown that, under some hypotheses on the solid flux $q_{b}$, it is hyperbolic, i.e. one can always find a complete set of eigenvectors. Moreover, it can be shown that the eigenvalues of the system are $u$ (whose multiplicity is, in the general case, equal to $n_{s}$ ), two external eigenvalues $S_{L}, S_{R}$, that may be approached by $u-\sqrt{g\left(1+R_{c}\right) h}, u+\sqrt{g\left(1+R_{c}\right) h}$, and a smaller internal eigenvalue $S_{M} \approx 0$. The eigenvalue $S_{M}$ is usually associated to the propagation of waves at the interface bed-plume, while $S_{L}$ and $S_{R}$ are identified with the velocity of waves at the interface plume-ambient water. Nevertheless, this is a rather simplified interpretation which is only valid for weak fluid-bed interactions and small Froude number. In general, surface waves move in tandem with the bed waves, so that bed and surface waves interact strongly and $S_{L}, S_{R}$ describe the propagation of both surface waves and bed waves and each of the celerities can not be identified solely with a surface wave or solely with a bed wave (see $[9,21,25]$ ).

As in [23], a splitting technique for erosion/deposition source terms will be used to approximate the solutions of system (2.8). At every time stage, first a finite volume method is applied to the homogeneous system:

$$
\partial_{t}\left(\begin{array}{c}
W \\
H
\end{array}\right)+\left(\begin{array}{c|c}
\mathcal{A}(W) & -S(W) \\
\hline 0 & 0
\end{array}\right) \partial_{x}\left(\begin{array}{c}
W \\
H
\end{array}\right)=0,
$$

and then the obtained cell values are updated by solving the ODE:

$$
\partial_{t} W=G(W) .
$$

As the numerical treatment of the second stage is the same than the one proposed in [23], we focus on the numerical solution of (2.14). For the sake of simplicity, we also consider $H=$ const. so that (2.14) may be rewritten in the form

$$
\partial_{t} W+\mathcal{A}(W) \partial_{x} W=0 .
$$

The numerical methods obtained in this article are easily adaptable to the general case in which $H$ is not constant. Further comments on this topic are included below.

As it is usual for shallow water models, the numerical scheme will be required to be exactly well-balanced for the water-at-rest solutions of (2.16). If the solid transport discharge formula is such that $u=0$ implies $q_{b}=0$ (what is the case for the Grass formula (2.3)), these stationary solutions are given by:

$$
u=0, \quad c=\text { const. }, \quad h+z_{b}=\text { const. }
$$

\section{Approximate Riemann solvers}

Let us consider a general nonconservative system

$$
\partial_{t} W+\mathcal{A}(W) \partial_{x} W=0, \quad x \in \mathbb{R}, t>0,
$$

where $W(x, t)$ belongs to $\Omega$, an open convex subset of $\mathbb{R}^{N}$, and $W \in \Omega \mapsto \mathcal{A}(W) \in \mathcal{M}_{N}(\mathbb{R})$ is a smooth locally bounded map. We suppose that system (3.1) is strictly hyperbolic, that is, for each $W \in \Omega$ matrix $\mathcal{A}(W)$ has $N$ real distinct eigenvalues $\lambda_{1}(W)<\ldots<\lambda_{N}(W)$, with associated eigenvectors $R_{1}(W), \ldots, R_{N}(W)$. We also suppose that for each $i=1, \ldots, N$, the characteristic field $R_{i}(W)$ is either genuinely nonlinear or linearly degenerate. 
In [28] a generalization of the definition of approximate Riemann solvers for nonconservative system was introduced. This definition is based on the choice of a family of paths, i.e. a family of Lipschitz continuous paths, $\Phi\left(s ; W_{L}, W_{R}\right), s \in[0,1]$, which must satisfy certain regularity and compatibility conditions, and in particular

$$
\Phi\left(0 ; W_{L}, W_{R}\right)=W_{L}, \quad \Phi\left(1 ; W_{L}, W_{R}\right)=W_{R}, \quad \forall W_{L}, W_{R} \in \Omega,
$$

and

$$
\Phi(s ; W, W)=W, \quad \forall s \in[0,1], W \in \Omega .
$$

We only consider here the particular case of simple solvers:

Definition 3.1. Let $\Phi$ be a family of paths in $\Omega$. Let us suppose that, for every pair of states $W_{L}$ and $W_{R} \in \Omega$, a finite number $m \geq 1$ of speeds

$$
\sigma_{0}=-\infty<\sigma_{1}<\ldots<\sigma_{m}<\sigma_{m+1}=+\infty
$$

and $m-1$ intermediate states

$$
W_{0}=W_{L}, W_{1}, \ldots, W_{m-1}, W_{m}=W_{R}
$$

are chosen in a continuous manner. The function $\widetilde{V}: \mathbb{R} \times \Omega \times \Omega \mapsto \Omega$ given by

$$
\widetilde{V}\left(v, W_{L}, W_{R}\right)=W_{j} \quad \text { if } \sigma_{j}<v<\sigma_{j+1},
$$

is said to be a $\Phi$-approximate Riemann solver for (3.1) if it satisfies

$$
\sum_{j=0}^{m-1} \sigma_{j+1}\left(W_{j+1}-W_{j}\right)=\int_{0}^{1} \mathcal{A}\left(\Phi\left(s ; W_{L}, W_{R}\right)\right) \frac{\partial \Phi}{\partial s}\left(s ; W_{L}, W_{R}\right) \mathrm{d} s .
$$

Any $\Phi$-approximate Riemann Solver for (3.1) leads to a numerical method which is is path-conservative in the sense introduced in [28]:

$$
W_{i}^{n+1}=W_{i}^{n}-\frac{\Delta t}{\Delta x}\left(D^{+}\left(W_{i-1}^{n}, W_{i}^{n}\right)+D^{-}\left(W_{i}^{n}, W_{i+1}^{n}\right)\right),
$$

where

$$
\begin{aligned}
& D^{-}\left(W_{L}, W_{R}\right)=-\int_{-\infty}^{0}\left(\widetilde{V}\left(v ; W_{L}, W_{R}\right)-W_{L}\right) \mathrm{d} v \\
& D^{+}\left(W_{L}, W_{R}\right)=-\int_{0}^{\infty}\left(\widetilde{V}\left(v ; W_{L}, W_{R}\right)-W_{R}\right) \mathrm{d} v
\end{aligned}
$$

or, equivalently,

$$
D^{-}\left(W_{L}, W_{R}\right)= \begin{cases}\int_{0}^{1} \mathcal{A}\left(\Phi\left(s ; W_{L}, W_{R}\right)\right) \frac{\partial \Phi}{\partial s}\left(s ; W_{L}, W_{R}\right) \mathrm{d} s & \text { if } \sigma_{m}<0, \\ \sum_{\sigma_{j+1}<0} \sigma_{j+1}\left(W_{j+1}-W_{j}\right) & \text { if } \sigma_{1}<0<\sigma_{m}, \\ 0 & \text { if } \sigma_{1}>0 .\end{cases}
$$




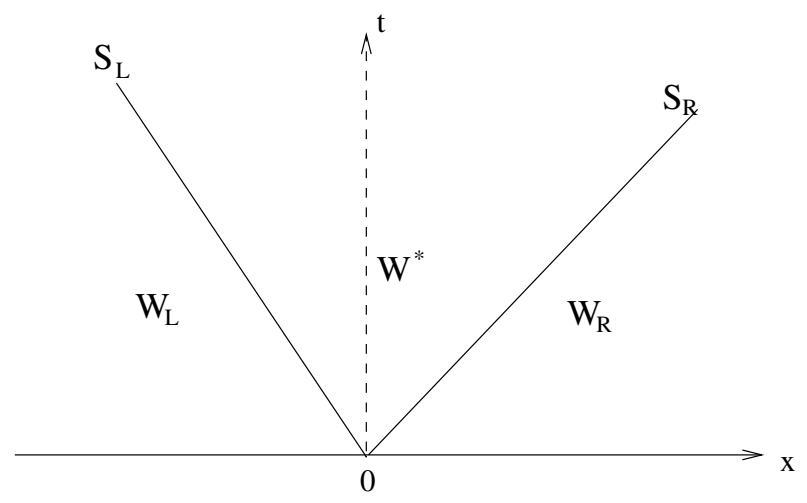

FiguRE 2. HLL solver.

$$
D^{+}\left(W_{L}, W_{R}\right)= \begin{cases}0 & \text { if } \sigma_{m}<0, \\ \sum_{\sigma_{j+1}>0} \sigma_{j+1}\left(W_{j+1}-W_{j}\right) & \text { if } \sigma_{1}<0<\sigma_{m}, \\ \int_{0}^{1} \mathcal{A}\left(\Phi\left(s ; W_{L}, W_{R}\right)\right) \frac{\partial \Phi}{\partial s}\left(s ; W_{L}, W_{R}\right) \mathrm{d} s & \text { if } \sigma_{1}>0 .\end{cases}
$$

The easiest example of $\Phi$-simple Riemann solver is obtained if $m=2$. In this case, the approximate Riemann solution consists of two waves of speed $\sigma_{1}=S_{L}, \sigma_{2}=S_{R}$ linking three constant states $W_{L}, W^{*}, W_{R}$ (see Fig. 2):

$$
\tilde{V}^{H L L}\left(v ; W_{L}, W_{R}\right)= \begin{cases}W_{L} & \text { if } v \leq S_{L} \\ W^{*} & \text { if } S_{L} \leq v \leq S_{R}, \\ W_{R} & \text { if } v \geq S_{R}\end{cases}
$$

The consistency condition (3.7), that reduces in this case to:

$$
S_{L}\left(W^{*}-W_{L}\right)+S_{R}\left(W_{R}-W^{*}\right)=\int_{0}^{1} \mathcal{A}\left(\Phi\left(s ; W_{L}, W_{R}\right)\right) \frac{\partial \Phi}{\partial s}\left(s ; W_{L}, W_{R}\right) \mathrm{d} s,
$$

allows us to determines the intermediate state:

$$
W^{*}=\frac{S_{R} W_{R}-S_{L} W_{L}-\int_{0}^{1} \mathcal{A}\left(\Phi\left(s ; W_{L}, W_{R}\right)\right) \partial_{s} \Phi\left(s ; W_{L}, W_{R}\right) \mathrm{d} s}{S_{R}-S_{L}} .
$$

The corresponding numerical scheme is given by (3.8), (3.11), (3.12) with $m=2, \sigma_{1}=S_{L}, \sigma_{2}=S_{R}$.

This is a natural extension of the HLL solver to nonconservative systems: if the system is conservative, the standard HLL solver is recovered regardless of the chosen family of paths. In the nonconservative case, different choices of the family of paths lead to different HLL solvers. This fact is related to the non-uniqueness of definition of weak solution for nonconservative system: according to the theory developed by Dal Maso et al. in [10] every choice of family of paths lead to a possible definition of weak solution, whose corresponding jump conditions are the following:

$$
\lambda[W]=\int_{0}^{1} \mathcal{A}\left(\Phi\left(s ; W^{-}, W^{+}\right)\right) \frac{\partial \Phi}{\partial s}\left(s ; W^{-}, W^{+}\right) \mathrm{d} s
$$


where $\lambda$ is the speed of propagation of the discontinuity and $[W]=W^{+}-W^{-}$. Therefore, Definition 3.1 is formally consistent with the definition of weak solution associated to the family $\Phi$.

As it was mentioned in the Introduction, the correct choice of the family of paths should be related to the viscous profile. But in most cases, as it happens with the turbidity current introduced in the previous Section, it is not even clear what is the correct parabolic regularization of the system. When there are no clear indications about the correct family of paths to be chosen, the family of straight segments

$$
\Psi\left(s ; W_{L}, W_{R}\right)=W_{L}+s\left(W_{R}-W_{L}\right),
$$

is a sensible choice, as their corresponding jump conditions are expected to give a third order approximation of the physically correct ones (see Appendix A).

Therefore, we shall consider here the family of straight segments (3.16) to define the weak solutions for the system (2.16). The corresponding jump conditions are the following:

$$
\left\{\begin{array}{l}
\lambda[h]=[q] \\
\lambda[q]=\left[\frac{q^{2}}{h}+\frac{g}{2}(1+R c) h^{2}\right]+g\left(\frac{h^{-}+h^{+}}{2}+R \frac{(h c)^{-}+(h c)^{+}}{2}\right)\left(z_{b}^{+}-z_{b}^{-}\right) \\
\lambda[h c]=[q c] \\
\lambda\left[z_{b}\right]=\left[q_{b}\right]
\end{array}\right.
$$

and the consistency condition (3.7) reduces to:

$$
\sum_{j=0}^{m-1} \sigma_{j+1}\left(W_{j+1}-W_{j}\right)=F\left(W_{R}\right)-F\left(W_{L}\right)+\mathcal{B}\left(W_{L}, W_{R}\right)
$$

where

$$
\mathcal{B}\left(W_{L}, W_{R}\right)=\left(g\left(\frac{h_{L}+h_{R}}{2}+R \frac{(h c)_{L}+(h c)_{R}}{2}\right)\left(z_{b R}-z_{b L}\right)\right) .
$$

Another reason to choose the family of paths (3.16) is related to the well-balanced property: a numerical method is said to be well-balanced for water at rest solutions if, when it is applied to an initial sequence of cell values $\left\{W_{i}^{0}\right\}$ that lie on the same curve (2.17), one has

$$
W_{i}^{n}=W_{i}^{0}, \quad \forall i, \forall n .
$$

It can be trivially checked that a numerical method (3.8) is well-balanced in this sense if, and only if, given two states $W_{L}$ and $W_{R}$ satisfying

$$
u_{L}=u_{R}=0, \quad c_{L}=c_{R}, \quad h_{L}+z_{b, L}=h_{R}+z_{b, R},
$$

one has

$$
D^{ \pm}\left(W_{L}, W_{R}\right)=0 .
$$

In the particular case of a method obtained from a $\Phi$-approximate Riemann solver, by adding (3.11) and (3.12) and taking into account (3.7), we deduce from (3.21):

$$
\int_{0}^{1} \mathcal{A}\left(\Phi\left(s ; W_{L}, W_{R}\right)\right) \frac{\partial \Phi}{\partial s}\left(s ; W_{L}, W_{R}\right) \mathrm{d} s=0 .
$$




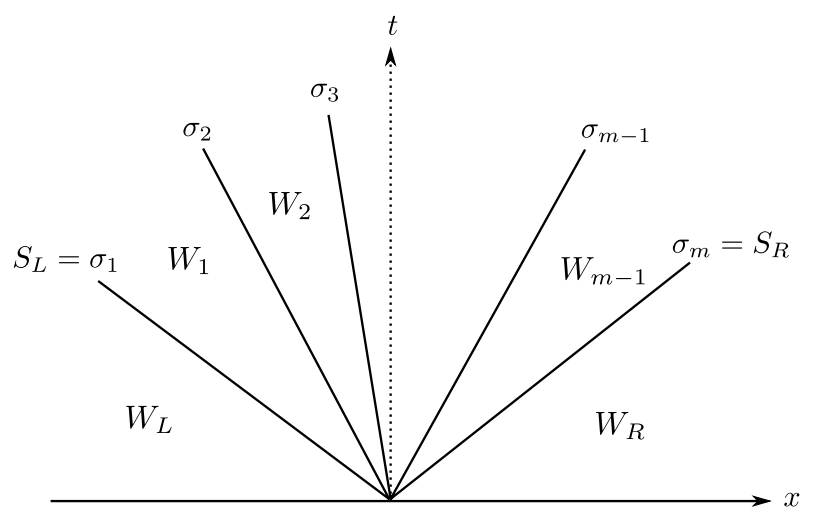

Figure 3. Sketch of a HLLC solver.

Therefore, in order to have the well-balanced property for water at rest solution, (3.22) has to be fulfilled for every pair of states satisfying (3.20) . The family of straight segments $\Psi$ satisfies this necessary condition: some straightforward calculations show that

$$
\int_{0}^{1} \mathcal{A}\left(\Psi\left(s ; W_{L}, W_{R}\right)\right) \frac{\partial \Psi}{\partial s}\left(s ; W_{L}, W_{R}\right) \mathrm{d} s=F\left(W_{R}\right)-F\left(W_{L}\right)+\mathcal{B}\left(W_{L}, W_{R}\right)=0
$$

if $W_{L}$ and $W_{R}$ satisfy $(3.20)$.

In general, (3.22) is not a sufficient condition to have the well-balanced property: for instance, the HLL solver based on the family $\Psi$ is not well-balanced for water at rest solutions.

From the expressions (3.9)-(3.10) of the fluctuations, it can be easily deduced that a sufficient condition to have the well-balanced property is the following: given two states $W_{L}$ and $W_{R}$ satisfying (3.20) the corresponding approximate Riemann solution is given by

$$
\widetilde{V}\left(v ; W_{L}, W_{R}\right)=\left\{\begin{array}{l}
W_{L} \text { if } v<0 \\
W_{R} \text { if } v>0
\end{array}\right.
$$

\section{HLLC SOLVER}

A HLLC Riemann solver can be considered as an extension of the HLL one in which, besides the two external waves of speed $\sigma_{1}=S_{L}$ and $\sigma_{m}=S_{R}, m-2$ internal waves of speeds $\sigma_{2}, \ldots, \sigma_{m-1}$ connecting $m-1$ intermediate states $W_{1}, \ldots, W_{m-1}$ are considered (see Fig. 3 ). Therefore, it is a $\Phi$-simple Riemann solver whose speeds and intermediate states are:

$$
\begin{gathered}
\sigma_{0}=-\infty<S_{L}=\sigma_{1}<\sigma_{2}<\cdots<\sigma_{m}=S_{R}<\sigma_{m+1}=\infty, \\
W_{0}=W_{L}, W_{1}, \ldots, W_{m-1}, W_{m}=W_{R} .
\end{gathered}
$$

If we assume that $S_{L}$ and $S_{R}$ are given by some estimates, then it is necessary to fix $(N+1)(m-1)-1$ scalars to determine the simple solver: $m-2$ speeds and $N(m-1)$ components of the intermediate states. Notice that the $N$ conditions given by (3.7) are enough to determine the solver only if $m=2$, i.e. in the HLL case. For $m>2$ some more conditions have to be taken into account in order to have the same number of unknowns and equations. To do this, the jump conditions (3.15) at every intermediate wave may be considered:

$$
\sigma_{j+1}\left(W_{j+1}-W_{j}\right)=\int_{0}^{1} \mathcal{A}\left(\Phi\left(s ; W_{j}, W_{j+1}\right) \partial_{s} \Phi\left(s ; W_{j}, W_{j+1}\right) \mathrm{d} s, \quad j=0, \ldots, m-1 .\right.
$$



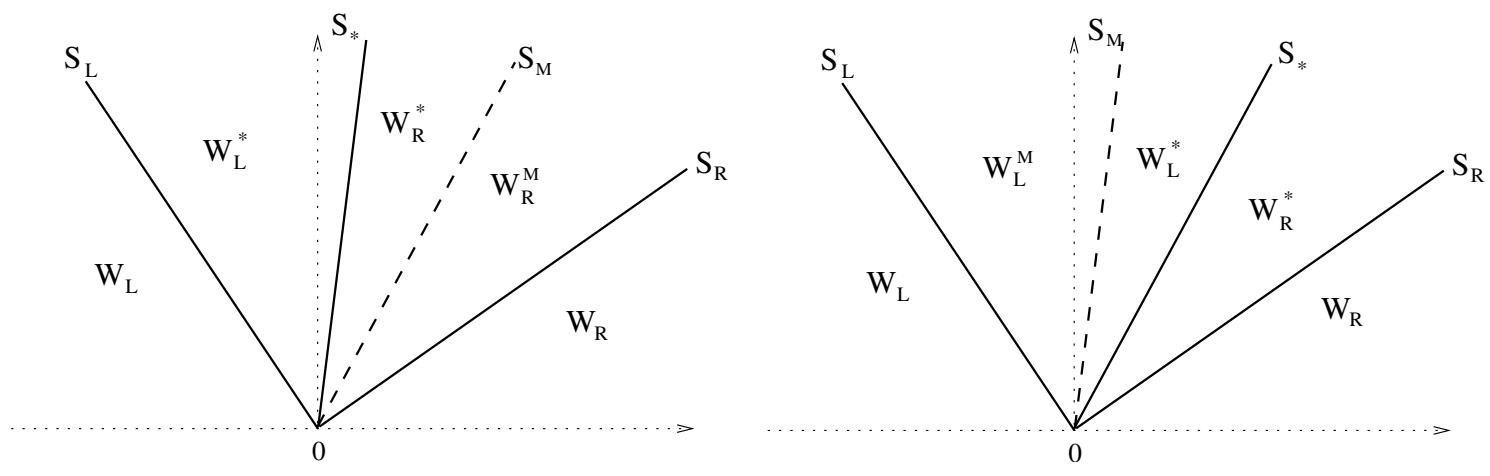

FiguRE 4. Waves configuration. Left: $S^{*}<S_{M}$. Right: $S_{M}<S^{*}$.

These jump conditions give us $m N$ more equations. In practice, $(N+1)(m-1)-1$ equations have to be chosen among the consistency condition (3.7) and the jump conditions (4.3). Let us remark that in order to have a $\Phi$-simple solver, if some of the components of the consistency condition (3.7) are not explicitly chosen, then it should be possible to recover it from the selected equations. Notice that a number of equations lower than $(N+1)(m-1)-1$ can be chosen and some of the unknowns may be then considered as free parameters what allows to obtain a family of HLLC solvers. Once the intermediate speeds and states have been calculated, the corresponding numerical scheme is given by (3.8), (3.11) and (3.12).

Let us apply this general principle to system (2.8). As it has been said, the erosion/deposition and the water entrainment source terms are neglected and $H$ is assumed to be constant. Two different HLLC solvers will be considered here consisting of four waves of speed $S_{L}, S_{M}, S^{*}, S_{R}$, linking five states:

$$
\left(W_{L}, W_{L}^{*}, W_{R}^{*}, W_{R}^{M}, W_{R}\right) \text { if } S^{*}<S_{M}
$$

or

$$
\left(W_{L}, W_{L}^{M}, W_{L}^{*}, W_{R}^{*}, W_{R}\right) \text { if } S_{M}<S^{*}
$$

(see Fig. 4).

Due to the expression of the $D^{ \pm}$functions given by (3.11)-(3.12), it is enough to define the simple Riemann solver when $S_{L}<0<S_{R}$. Only the case $S^{*}>S_{M}$, will be considered, the case $S^{*}<S_{M}$ being analogous. In order to define the solver, 16 scalars have to be fixed: the wave speeds $S_{L}, S_{R}, S^{*}, S_{M}$ and the components of the intermediate states $W_{L}^{*}, W_{R}^{*}, W_{L}^{M}$. The components of the intermediate states will be denoted as follows:

$$
W_{L}=\left[\begin{array}{c}
h_{L} \\
h_{L} u_{L} \\
h_{L} c_{L} \\
z_{b L}
\end{array}\right], \quad W_{L}^{M}=\left[\begin{array}{c}
h_{L}^{M} \\
h_{L}^{M} u_{L}^{M} \\
h_{L}^{M} c_{L}^{M} \\
z_{b L}^{M}
\end{array}\right], \quad W_{L}^{*}=\left[\begin{array}{c}
h_{L}^{*} \\
h_{L}^{*} u_{L}^{*} \\
h_{L}^{*} c_{L}^{*} \\
z_{b L}^{*}
\end{array}\right], \quad W_{R}^{*}=\left[\begin{array}{c}
h_{R}^{*} \\
h_{R}^{*} u_{R}^{*} \\
h_{R}^{*} c_{R}^{*} \\
z_{b R}^{*}
\end{array}\right], \quad W_{R}=\left[\begin{array}{c}
h_{R} \\
h_{R} u_{R} \\
h_{R} c_{R} \\
z_{b R}
\end{array}\right] .
$$




\subsection{An essentially three-wave HLLC solver}

We consider the following nonlinear system composed by the first, third, and fourth components of the jump conditions (3.17) for the four waves speeds, and the second component of the consistency condition (3.7):

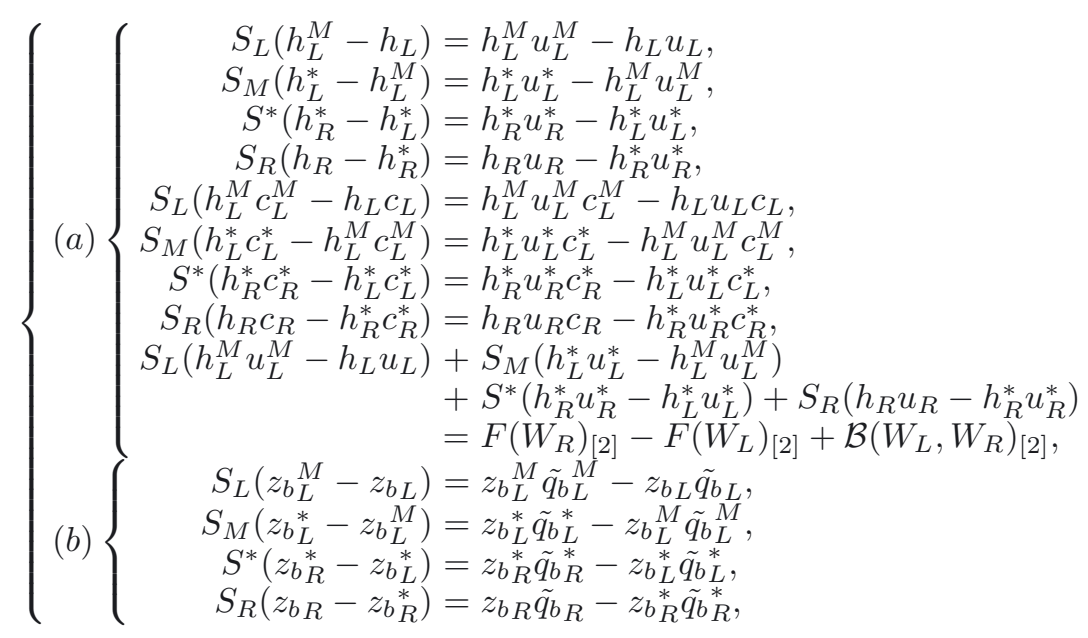

where $\tilde{q}_{b}, \tilde{q}_{b}^{*}, \tilde{q}_{b}^{*}, \tilde{q}_{b_{R}}$ stand for the solid transport flux $\tilde{q}_{b}$ evaluated in the corresponding state, $F$ is given by $(2.10), \mathcal{B}\left(W_{L}, W_{R}\right)$ is defined by (3.19), and the subscript [2] denotes the second component of these vectors. It can be easily checked that the first component of the consistency condition (3.7) is recovered by adding the first four equations, the third component is recovered by adding equations 5th to 9th, and the fourth one by adding the four last equations.

We have thus 13 equations for 16 unknowns. If we fix the value of $h_{L}^{M}$ by:

$$
h_{L}^{M}=h_{L}^{*}
$$

and

$$
\begin{aligned}
& S_{L}=u_{L}-\frac{k_{L}}{h_{L}}, \\
& S_{R}=u_{R}+\frac{k_{R}}{h_{R}},
\end{aligned}
$$

where $k_{L}$ and $k_{R}$ are two positive values, then a solution of system (4.4)-(a) is given by

$$
\begin{gathered}
u_{L}^{M}=u_{L}^{*}, \quad c_{L}^{M}=c_{L}^{*}, \\
S^{*}=u_{L}^{*}=u_{R}^{*}=\frac{k_{L} u_{L}+k_{R} u_{R}-\mathcal{P}_{L R}}{k_{L}+k_{R}}, \\
\frac{1}{h_{L}^{*}}=\frac{1}{h_{L}}+\frac{k_{R}\left(u_{R}-u_{L}\right)-\mathcal{P}_{L R}}{k_{L}\left(k_{L}+k_{R}\right)}, \\
\frac{1}{h_{R}^{*}}=\frac{1}{h_{R}}+\frac{k_{L}\left(u_{R}-u_{L}\right)+\mathcal{P}_{L R}}{k_{R}\left(k_{L}+k_{R}\right)},
\end{gathered}
$$

where

$$
\mathcal{P}_{L R}=\frac{1}{2} g\left(1+R c_{R}\right) h_{R}^{2}-\frac{1}{2} g\left(1+R c_{L}\right) h_{L}^{2}+\mathcal{B}\left(W_{L}, W_{R}\right)_{[2]} .
$$


The subsystem (4.4)-(b) has still to be solved. Two different situations are considered:

- Steady bottom: if $\widetilde{q_{b}}=0$, the solution is given by

$$
S_{M}=0, \quad z_{b L}^{M}=z_{b L}, \quad z_{b L}^{*}=z_{b R}^{*}=z_{b R} .
$$

- Moving bottom: in this case, the solution is given by

Remark 4.1. Observe that:

$$
\begin{aligned}
z_{b}^{M} & =\frac{u_{L}-\frac{k_{L}}{h_{L}}-\tilde{q_{b}}}{u_{L}-\frac{k_{L}}{h_{L}}-{\tilde{q_{b}}}_{L}^{*}} z_{b L}, \\
z_{b_{R}}^{*} & =\frac{u_{R}+\frac{k_{R}}{h_{R}}-\tilde{q_{b R}}}{u_{R}+\frac{k_{R}}{h_{R}}-{\tilde{q_{b}}}_{R}^{*}} z_{b_{R}}, \\
z_{b_{L}}^{*} & =\frac{S^{*}-{\tilde{q_{b}}}^{*}}{S^{*}-{\tilde{q_{b}}}_{L}^{*}} z_{b_{R}^{*}}^{*}, \\
S_{M} & ={\tilde{q_{b}}}_{L}^{*} \cdot
\end{aligned}
$$

(1) the variables $h$ and $u$ are constant across the wave associated to $S_{M}$. Therefore, the obtained HLLC solver has only three waves for the hydrodynamical variables;

(2) in the case of a steady bottom, the topography is only discontinuous across the wave of speed $S_{M}=0$, and the source term only contributes to this stationary wave;

(3) in the case of a moving bottom, $S_{L}$ and $S_{R}$ correspond to the external waves while $S_{M}$ is expected to be an approximation of the internal one. Remark that $z_{b}$ jumps at each of the waves which is in connection to what has been said in Section 2 regarding the interaction between fluid and topography. Notice that, if $\widetilde{q_{b}}$ is small compared to the external wave speeds $S_{L}, S_{R}$, then one has $z_{b_{L}^{*}}^{*} \approx z_{b_{L}}$ and $z_{b_{R}}^{*} \approx z_{b_{R}}$ what is in agreement with the interpretation of $S_{M}$ as the speed of waves of the sediment layer for weak interactions;

(4) the sediment concentration $c$ is only discontinuous across the wave of speed $S^{*}$. This speed coincide with the fluid velocity at the intermediate region $u_{L}^{*}=u_{R}^{*}$.

Remark 4.2. Note that if $R=0$, system (2.8) coincides with the shallow-water equations with pollutant concentration.

(a) This HLLC solver is different from the one introduced in [32]: while in the latter the equality $h_{L}^{*}=h_{R}^{*}$ is assumed, this is not the case here;

(b) if the bottom is steady and flat, the HLLC solver coincides with the Suliciu relaxation method presented in [3]. Therefore, it is entropy satisfying.

Let us introduce the following notation:

$$
p(h, c)=\frac{g}{2}(1+R c) h^{2}, \quad \alpha=3 / 2 .
$$

Following the techniques introduced in [3] the following result can be proved (see Appendix B).

Theorem 4.3. The numerical scheme based on the HLLC solver based on the definitions (4.5)-(4.8) with $k_{L}$ and $k_{R}$ given by:

$$
\text { if } \mathcal{P}_{L R} \geq 0, \quad\left\{\begin{array}{l}
\frac{k_{L}}{h_{L}}=\sqrt{\frac{\partial p}{\partial h}\left(h_{L}, c_{L}\right)}+\alpha\left(\frac{\mathcal{P}_{L R}}{h_{R} \sqrt{\frac{\partial p}{\partial h}\left(h_{R}, c_{R}\right)}+u_{L}-u_{R}}\right)_{+} \\
\frac{k_{R}}{h_{R}}=\sqrt{\frac{\partial p}{\partial h}\left(h_{R}, c_{R}\right)}+\alpha\left(\frac{-\mathcal{P}_{L R}}{k_{L}}+u_{L}-u_{R}\right)_{+},
\end{array}\right.
$$




$$
\text { if } \mathcal{P}_{L R} \leq 0, \quad\left\{\begin{array}{l}
\frac{k_{R}}{h_{R}}=\sqrt{\frac{\partial p}{\partial h}\left(h_{R}, c_{R}\right)}+\alpha\left(\frac{-\mathcal{P}_{L R}}{h_{L} \sqrt{\frac{\partial p}{\partial h}\left(h_{L}, c_{L}\right)}+u_{L}-u_{R}}\right)_{+} \\
\frac{k_{L}}{h_{L}}=\sqrt{\frac{\partial p}{\partial h}\left(h_{L}, c_{L}\right)}+\alpha\left(\frac{\mathcal{P}_{L R}}{k_{R}}+u_{L}-u_{R}\right)_{+},
\end{array}\right.
$$

preserves the non-negativity of $h$.

In the case of a moving bottom, the analysis of the non-negativity preservation of $z_{b}$ is not an easy task, especially for complex expressions of $\widetilde{q_{b}}$. Nevertheless, for some simple formulae (for instance, the Grass model) it can be proved that the numerical scheme is non-negativity preserving for $z_{b}$ provided that $k_{L}$ and $k_{R}$ are large enough (see Appendix C).

Proposition 4.4. Suppose that $\widetilde{q_{b}}$ only depends on the variable $u$ that is, there exists some continuous function $\phi$ such that $\tilde{q_{b}}(h, u, c)=\phi(u)$. Then if $z_{b L}$ and $z_{b R}$ are non-negative and $k_{L}, k_{R}>0$ are large enough, the values $z_{b}^{M}, z_{b}^{*}, z_{b}^{*}$ defined by (4.8) are also non-negative.

Remark that this result is compatible with Theorem 4.3 in the sense that both the non-negativity of $h$ and $z$ can be preserved: in effect, the values of $k_{L}$ and $k_{R}$ given by (4.10)-(4.11) can take arbitrarily large values: see the proof in Appendix B.

Let us check finally that this HLLC solver is well-balanced for water at rest solutions. Given two states $W_{L}=\left[h_{L}, 0, c_{L} h_{L}, z_{b L}\right]^{T}, W_{R}=\left[h_{R}, 0, c_{R} h_{R}, z_{b R}\right]^{T}$ such that:

$$
c_{L}=c_{R}=\bar{c}, \quad h_{L}+z_{b L}=h_{R}+z_{b R}
$$

the following equalities can be easily checked:

$$
S_{M}=S^{*}=0, \quad W_{L}^{M}=W_{L}, \quad W_{R}^{*}=W_{R}, \quad W_{L}^{*}=\left[h_{L}, 0, \bar{c} h_{L}, z_{R}\right]^{T},
$$

but notice that the region in which the approximate solution takes the value $W_{L}^{*}$ disappears. Therefore, the approximate Riemann solver reduces to (3.24) so that the numerical scheme is well-balanced.

In practice:

- the speed of the fastest waves are given by (4.5) and Theorem 4.3;

- the stability requirement is $C F L \in(0,1]$, where the $C F L$ parameter is given by:

$$
C F L=\max _{i}\left\{\max \left(\left|S_{L, i+1 / 2}\right|,\left|S_{R, i+1 / 2}\right|\right)\right\} \frac{\Delta t}{\Delta x},
$$

where $\Delta x$ is the space step (which is assumed to be constant), $\Delta t$ it the time step, and $S_{L, i+1 / 2}, S_{R, i+1 / 2}$ are the fastest waves considered in the approximate Riemann solver at the intercell $x_{i+1 / 2}$.

Remark 4.5. If the depth of the non-erodible bottom, $H$, is not constant, a new wave of speed 0 is added through which only the variable $H$ is discontinuous. The system to be solved in order to compute the intermediate speeds is still (4.4) with only a modification in the 9th equation that now reads as follows:

$$
\begin{aligned}
S_{L}\left(h_{L}^{M} u_{L}^{M}-h_{L} u_{L}\right)+S_{M}\left(h_{L}^{*} u_{L}^{*}-h_{L}^{M} u_{L}^{M}\right)+S^{*}\left(h_{R}^{*} u_{R}^{*}-h_{L}^{*} u_{L}^{*}\right)+S_{R}\left(h_{R} u_{R}-h_{R}^{*} u_{R}^{*}\right) \\
=F\left(W_{R}\right)_{[2]}-F\left(W_{L}\right)_{[2]}+\mathcal{B}\left(\widetilde{W}_{L}, \widetilde{W}_{R}\right)_{[2]},
\end{aligned}
$$


where $\widetilde{W}=[W, H]^{T}$, and

$$
\mathcal{B}\left(\widetilde{W}_{L}, \widetilde{W}_{R}\right)=\left(g\left(\frac{h_{L}+h_{R}}{2}+R \frac{(h c)_{L}+(h c)_{R}}{2}\right)\left(z_{b R}-z_{b L}-\left(H_{R}-H_{L}\right)\right)\right.
$$

The definitions of the wave speeds and intermediate states remain the same with the only difference that now

$$
\mathcal{P}_{L R}=\frac{1}{2} g\left(1+R c_{R}\right) h_{R}^{2}-\frac{1}{2} g\left(1+R c_{R}\right) h_{R}^{2}+\mathcal{B}\left(\widetilde{W}_{L}, \widetilde{W}_{R}\right)_{[2]} .
$$

\subsection{A four-wave HLLC solver}

Let us consider now the system given by (4.4)-(a) and the following modification of (4.4)-(b):

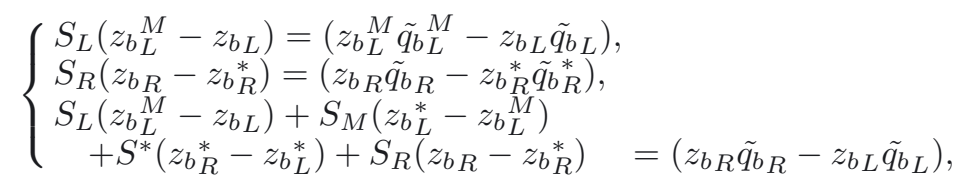

where the third components of the jump conditions at the waves of speed $S^{*}$ and $S^{M}$ are replaced by the third component of the consistency condition. We have now 12 equations and 16 unknowns. If $S_{L}, S_{R}$ are given by some estimates of the fastest wave speeds, $h_{L}^{M}$ and $S_{M}$ are defined by:

$$
\begin{gathered}
h_{L}^{M}=h_{L}^{*}+z_{b R}-z_{b L} . \\
S_{M}= \begin{cases}0 & \text { if } q_{b}=0 \\
\lambda_{\text {int }}\left(W_{0}\right) & \text { otherwise }\end{cases}
\end{gathered}
$$

where $\lambda_{\text {int }}\left(W_{0}\right)$ is the internal eigenvalue computed at an intermediate state $W_{1 / 2}$, a solution of the system is given by:

$$
\begin{gathered}
c_{R}^{*}=c_{R}, \quad c_{L}^{*}=c_{L}, \quad c_{L}^{M}=c_{L}, \\
S^{*}=\frac{h_{R} u_{R}\left(S_{R}-u_{R}\right)+h_{L} u_{L}\left(u_{L}-S_{L}\right)+S_{M}\left(S_{L}-S_{M}\right)\left(z_{b R}-z_{b L}\right)-\mathcal{P}_{L R}}{h_{R}\left(S_{R}-u_{R}\right)+h_{L}\left(u_{L}-S_{L}\right)+\left(S_{L}-S_{M}\right)\left(z_{b R}-z_{b L}\right)} \\
u_{L}^{*}=u_{R}^{*}=S^{*}, \\
u_{L}^{M}=\frac{h_{L} u_{L}+S_{L}\left(h_{L}^{M}-h_{L}\right)}{h_{L}^{M}}, \\
h_{L}^{*}=\frac{h_{L}\left(u_{L}-S_{L}\right)+\left(S_{L}-S_{M}\right)\left(z_{b R}-z_{b L}\right)}{S^{*}-S_{L}}, \\
h_{R}^{*}=\frac{h_{R}\left(u_{R}-S_{R}\right)}{S^{*}-S_{R}}, \\
z_{b L}^{M}=\frac{z_{b L}\left(-S_{L}+\tilde{q}_{b L}\right)}{\tilde{q}_{b L}^{M}-S_{L}}=\frac{z_{b R}\left(S_{R}-\tilde{q}_{b R}\right)}{S_{R}-\tilde{q}_{b R}^{*}}, \\
z_{b L}^{*}=\frac{S_{L}\left(z_{b L}-z_{b L}^{M}\right)+S_{R}\left(z_{b R}^{*}-z_{b R}\right)+z_{b R} \tilde{q}_{b R}-z_{b L} \tilde{q}_{b L}+S_{M} z_{b L}^{M}-S^{*} z_{b R}^{*}}{S_{M}-S^{*}} .
\end{gathered}
$$


Remark 4.6. The main difference between this HLLC solver and the one introduced in Section 4.1 is that $S_{M}$ explicitly appears in the definition of the hydrodynamic variables. If $\tilde{q_{b}}=0$, the two solvers coincide.

Concerning the non-negativity-preserving property of the four-wave HLLC, if $\tilde{q_{b}}=0$, the following result can be shown (see Appendix D).

Proposition 4.7. Assume that $S_{L}<S_{M}=0<S^{*}$ and

$$
\begin{aligned}
0<h_{L}^{M} \leq \max \left(\frac{h_{L}\left(S_{L}-u_{L}\right)}{s_{L}},\right. & \\
& \left.\frac{h_{R}\left(u_{R}-S_{R}\right)^{2}+h_{L}\left(u_{L}-S_{L}\right)^{2}+h_{L} u_{L}\left(S_{R}+2 S_{L}-2 u_{L}\right)-h_{L} S_{L} S_{R}-\mathcal{P}_{L R}}{-S_{L}\left(S_{R}-S_{L}\right)}\right) .
\end{aligned}
$$

Then, the HLLC scheme is non-negativity preserving and well defined, that is, if $h_{L} \geq 0$ and $h_{R} \geq 0$, then $h_{L}^{*} \geq 0, h_{R}^{*} \geq 0$ and $S^{*}<S_{R}$.

Remark 4.8. In general, the difficulty in (4.25) is to prove that the upper bound is positive. In fact, this is not possible when 'big steps' are present in the bottom. In that case, the essentially three-wave HLLC scheme should be used to ensure the non-negativity.

Finally, let us check the well-balanced property of the numerical scheme given by this HLLC-solver. Given two states $W_{L}=\left[h_{L}, 0, c_{L} h_{L}, z_{b L}\right]^{T}, W_{R}=\left[h_{R}, 0, c_{R} h_{R}, z_{b R}\right]^{T}$ such that:

$$
c_{L}=c_{R}=\bar{c}, \quad h_{L}+z_{b L}=h_{R}+z_{b R}
$$

the following equalities can be easily checked:

$$
S_{M}=S^{*}=0, \quad W_{L}^{*}=W_{R}^{*}=W_{R}, \quad W_{L}^{M}=W_{L},
$$

and the result follows.

\section{NUMERICAL TESTS}

The HLLC solvers introduced in Sections 4.1 and 4.2 will be named hereafter as E3W-HLLC and 4W-HLLC, respectively. In this section, we compare the numerical solutions provided by these two solvers between them and also with those provided by the Roe scheme introduced in [23] for some $1 \mathrm{~d}$ and $2 \mathrm{~d}$ test problems. The $C F L$ parameter is set to 0.9 .

\subsection{Well-balanced property}

From the definitions of E3W-HLLC and 4W-HLLC it follows that both schemes are well-balanced for steadystate solutions (2.17). Let us verify numerically this property by considering the following initial condition

$$
z_{b}(x, 0)=0.1 \mathrm{e}^{-(x-5.0)^{2}}, \quad h+z_{b}=1, \quad c(x, 0)=0.05, \quad q=0 .
$$

The numerical solution at $t=1$ is computed with 100 points in the interval $[0,5]$. The $L^{\infty}$ error for both schemes is shown in Table 1. 
TABLE 1. $L^{\infty}$ error at $t=1$ for E3W-HLLC and $4 \mathrm{~W}$-HLLC.

\begin{tabular}{|c|c|c|}
\hline & E3W-HLLC & 4W-HLLC \\
\hline$h$ & $1.514011 \mathrm{e}-12$ & $1.513789 \mathrm{e}-12$ \\
\hline$q$ & $1.954471 \mathrm{e}-13$ & $1.965307 \mathrm{e}-13$ \\
\hline$z$ & $3.246500 \mathrm{e}-13$ & $3.246153 \mathrm{e}-13$ \\
\hline
\end{tabular}

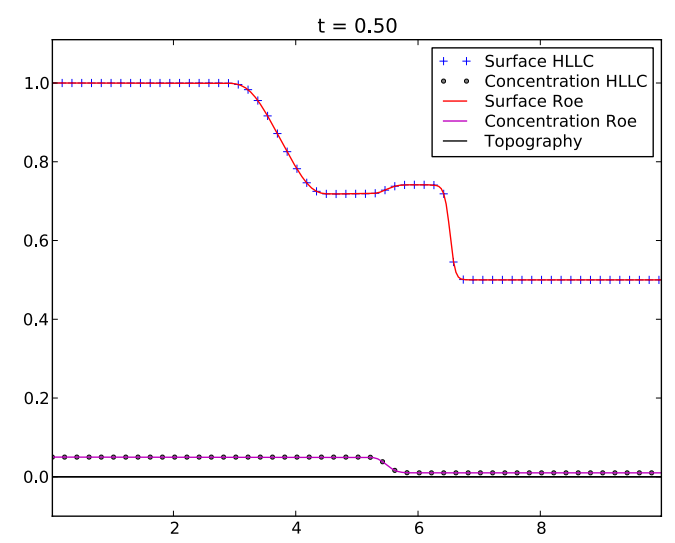

(a)

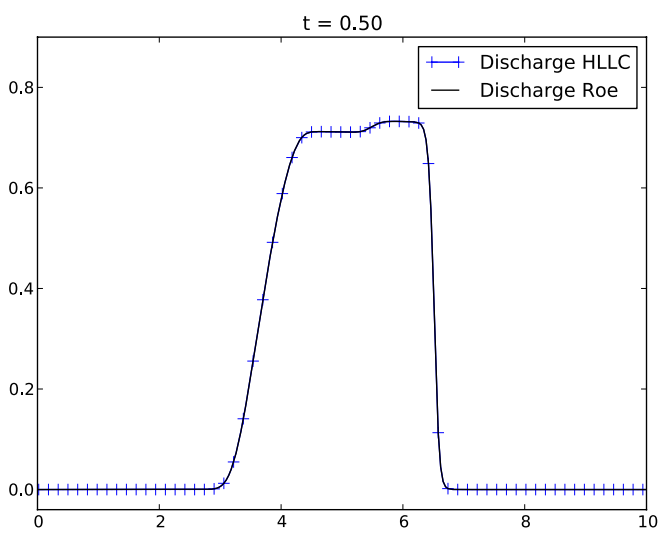

(b)

FIgURE 5. Simulation over flat topography.

\subsection{Flow over a flat bottom}

We consider $q_{b}=0$ and $z_{b}=$ const. The computational domain is the interval $[0,10]$ and the initial conditions are given by:

$$
h(x, 0)=\left\{\begin{array}{ll}
1 & \text { if } x<5, \\
0.5 & \text { if } x \geq 5,
\end{array} \quad c(x, 0)= \begin{cases}0.05 & \text { if } x<5 \\
0.01 & \text { if } x \geq 5\end{cases}\right.
$$

and $q(x, 0)=0$ for all $x \in[0,10]$. Free boundary conditions at both extremes of the interval are imposed by using a ghost-cell technique. In this case E3W-HLLC and 4W-HLLC coincide (see Rem. 4.6). The results are shown in Figure 5.

Let us see that no dog-leg effects appear at the sonic point in the numerical solutions provided by the HLLC solvers: it is not necessary to add any entropy-fix technique as it is the case for the Roe method. To see this, we consider the above described test problem with data:

$$
h(x, 0)= \begin{cases}2 & \text { if } x<5 \\ 0.1 & \text { if } x \geq 5\end{cases}
$$

The solutions obtained with both HLLC solvers and with the Roe method without an entropy-fix technique are compared in Figure 6.

\subsection{Transcritical flow}

We consider next a transcritical flow over a bump. The equations are solved in the interval $[0,25]$ with initial condition:

$$
h(x, 0)=0.33, \quad q(x, 0)=0.18, \quad c(x, 0)=0 .
$$




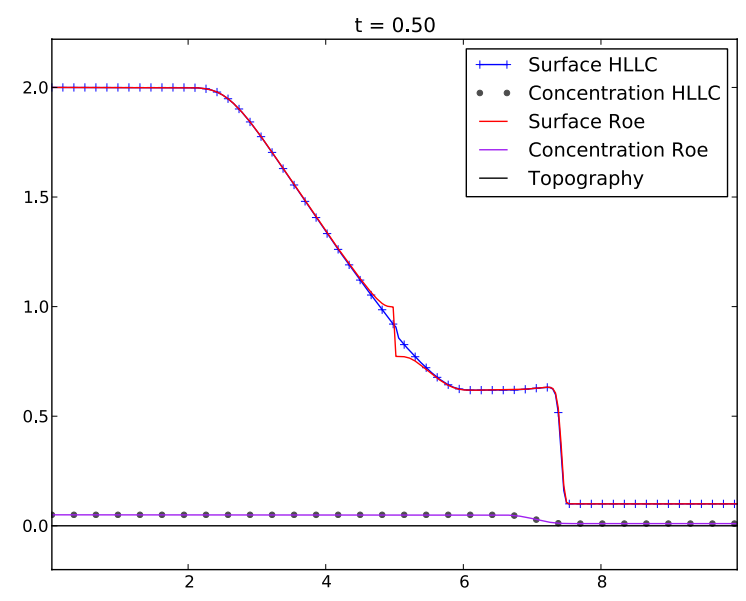

FiguRE 6. Entropy fix is needed for Roe scheme but not for HLLC.

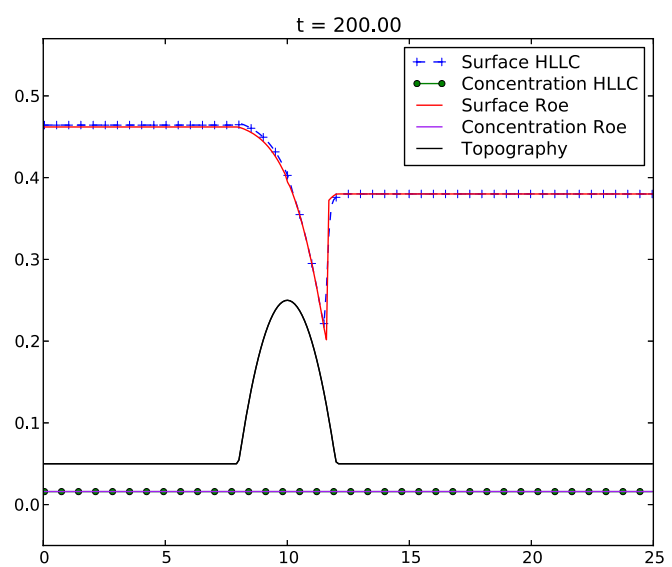

(a)

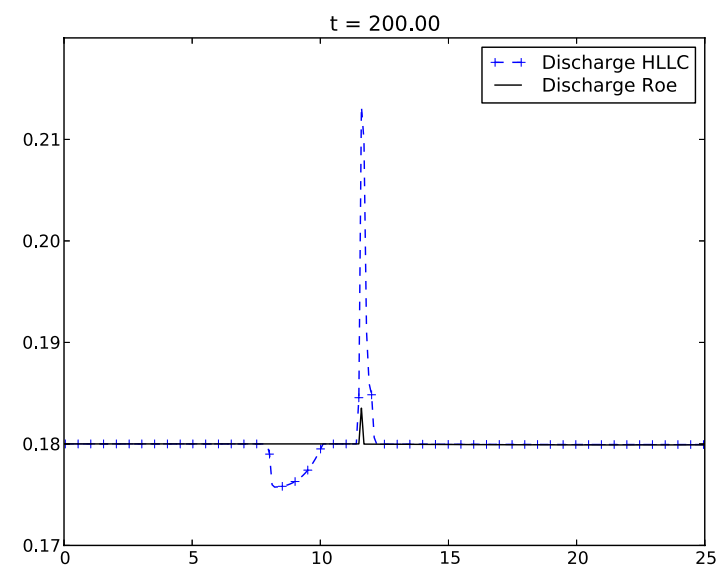

(b)

FiguRE 7. Transcritical flow. E3W-HLLC solver.

Again, $q_{b}=0$ and the bottom is given by:

$$
z_{b}(x)= \begin{cases}0.25-0.05(x-10)^{2} & \text { if } 8<x<12 \\ 0.05 & \text { otherwise. }\end{cases}
$$

The boundary conditions are $q(0, t)=0.18, h(25, t)=0.33$, and $c(0, t)=0.02$. Figures 7 and 8 show the steadystate reached using 250 points in the interval. Notice that, while the free surface and concentration (left plot in Figs. 7 and 8) computed with E3W-HLLC and 4W-HLLC are practically identical, the computed discharge $q$ is more accurate for the latter (right plot in Figs. 7 and 8). Notice that the Roe method gives a better result here, specially near the shock. This is due to the fact that the Roe method is less diffusive than the HLLC schemes. Nevertheless, the results are comparable. 


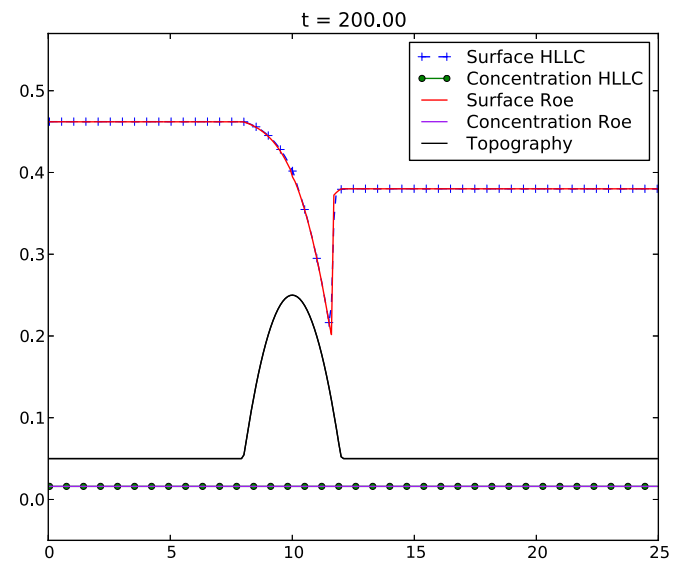

(a)

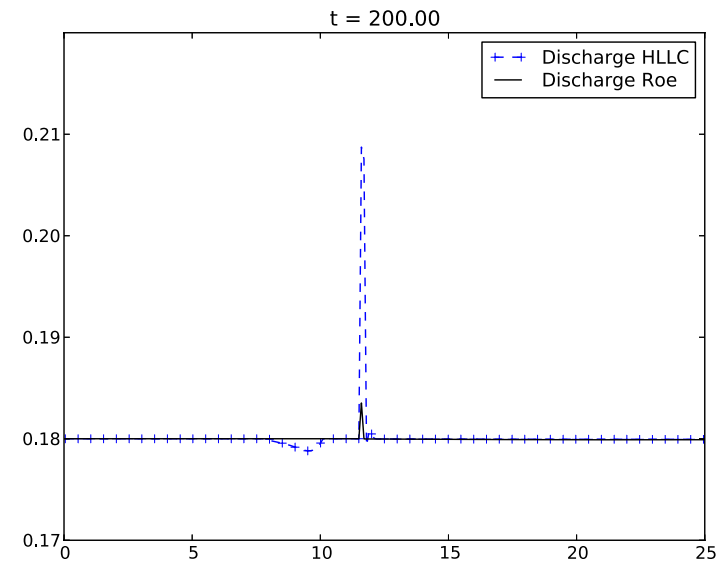

(b)

FiguRE 8. Transcritical flow. E3W-HLLC solver.

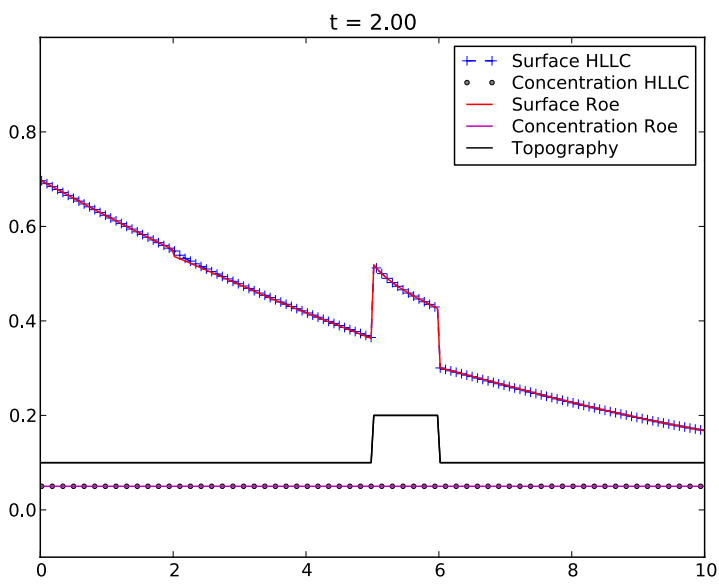

(a)

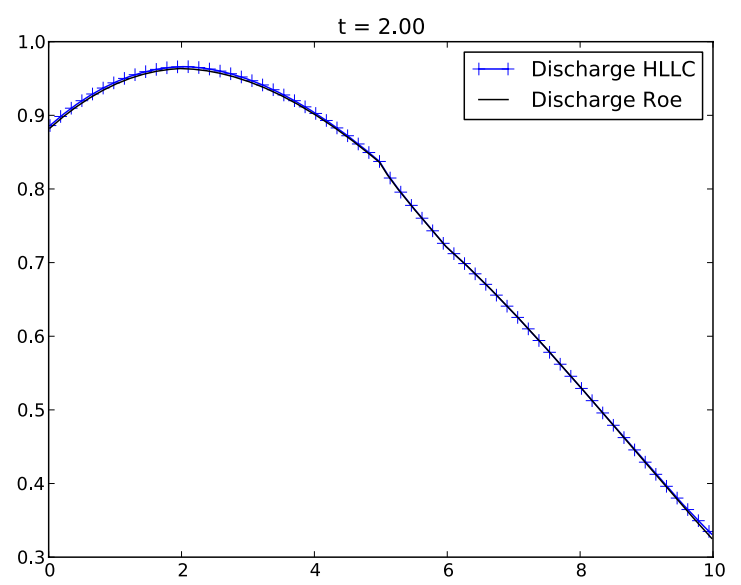

(b)

FiguRE 9. Dam-break problem. E3W-HLLC solver.

\subsection{A dam-break test case}

Let us consider now a dam-break problem over a bottom with a step. The equations are solved in the interval $[0,10]$ with initial condition:

$$
h(x, 0)=\left\{\begin{array}{lc}
1 & \text { if } x<2, \\
0 & \text { otherwise }
\end{array}, \quad c(x, 0)= \begin{cases}0.05 & \text { if } x<2 \\
0 & \text { otherwise }\end{cases}\right.
$$

and $q(x, 0)=0$, for all $x \in[0,10]$. We consider again $q_{b}=0$ and a fixed bottom given by:

$$
z_{b}(x)= \begin{cases}0.2 & \text { if } 5<x<6 \\ 0.1 & \text { otherwise }\end{cases}
$$




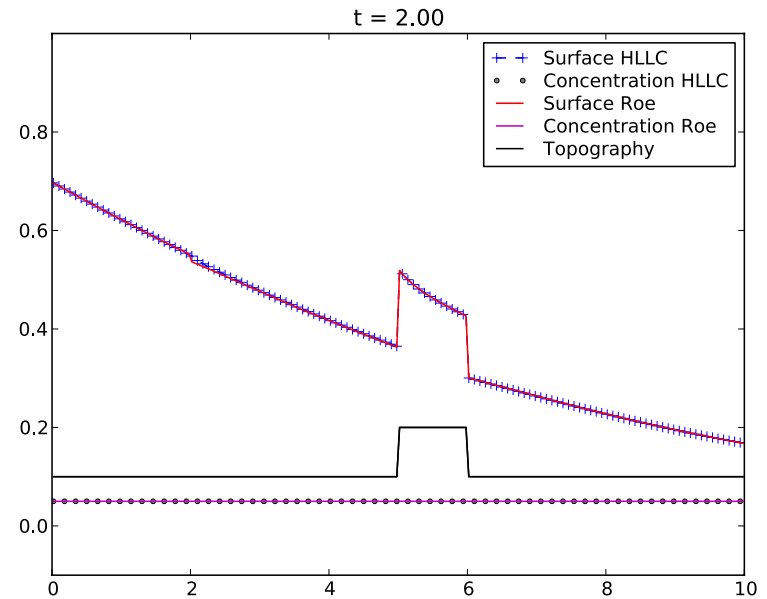

(a)

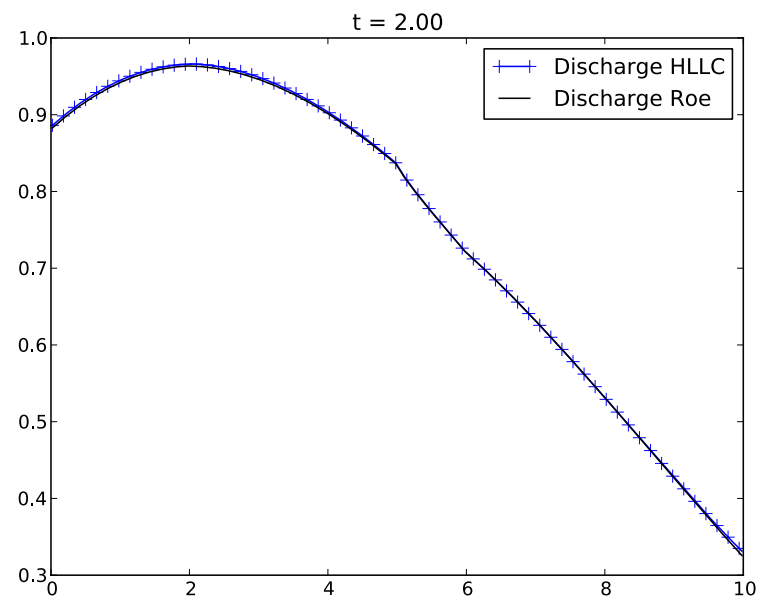

(b)

FiguRE 10. Dam-break problem. 4W- HLLC solver.

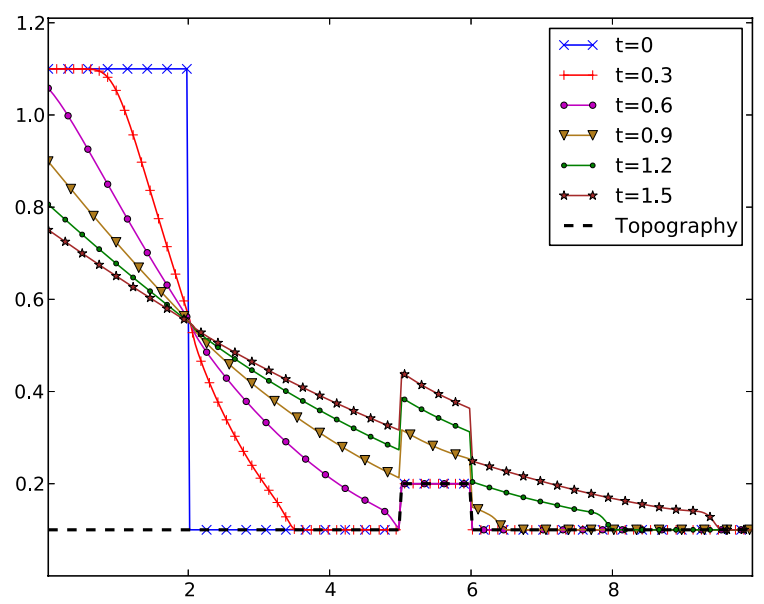

FIGURE 11. Surface evolution with E3W-HLLC solver.

Free boundary conditions are considered. The results are shown in Figures 9 and 10 for time $t=2$. In this test both HLLC schemes give similar results. In Figure 11 we can observe the surface evolution for different times.

\subsection{Moving bottom}

In this test, Grass formula $(2.4)-(2.5)$ is used with $A=0.005$. The interval is $[0,10]$ and the initial condition is given by

$$
(h+z)(x, 0)=1.1, \quad q(x, 0)=0, \quad c(x, 0)=0, \quad \forall x \in[0,10],
$$

and

$$
z_{b}(x, 0)= \begin{cases}0.2 & \text { if } 4<x<6 \\ 0.1 & \text { otherwise }\end{cases}
$$




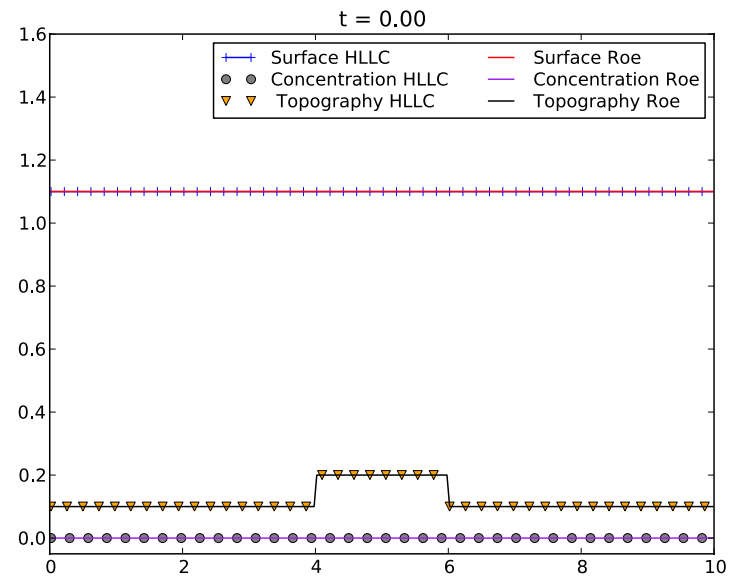

(a)

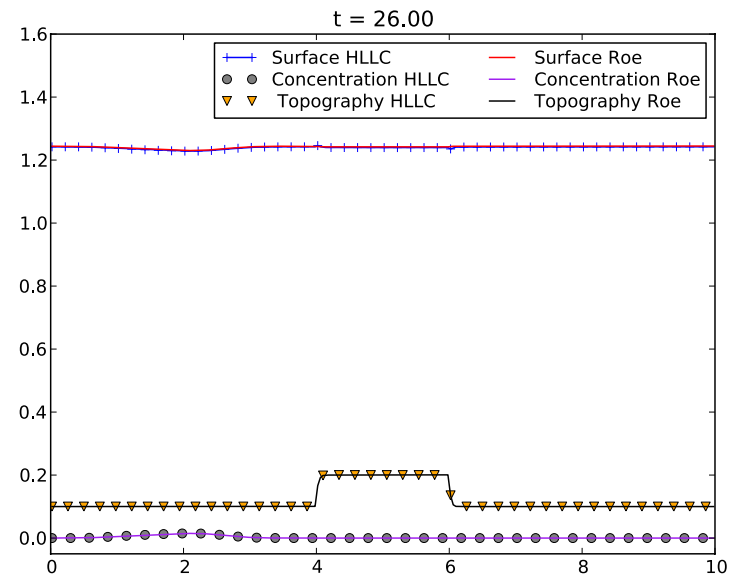

(b)

FIGURE 12. Moving bottom: E3W-HLLC.

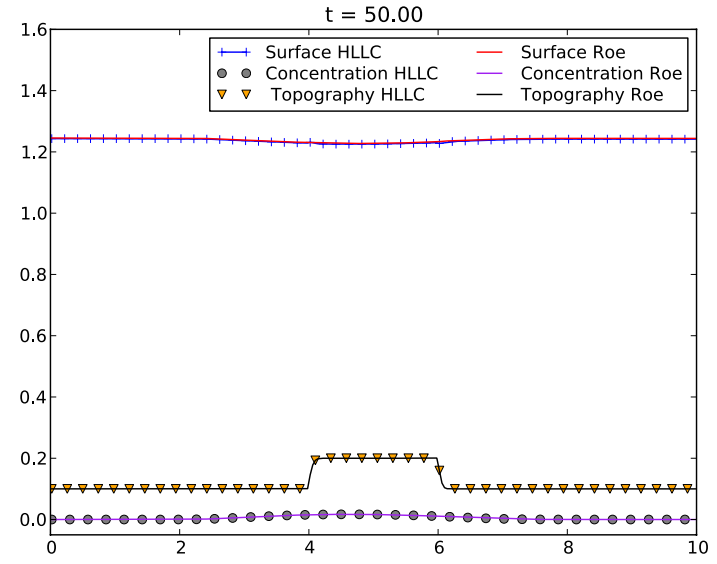

(a)

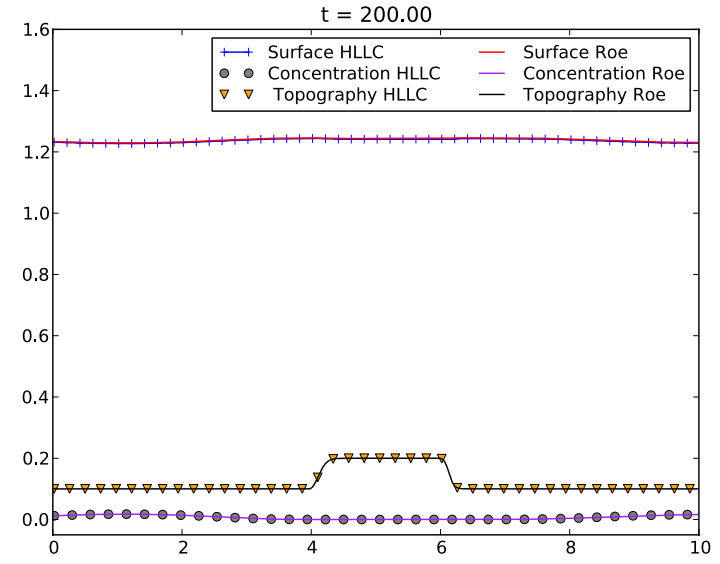

(b)

FiguRE 13. Moving bottom: E3W-HLLC.

We impose the boundary conditions $q(0, t)=0.5$ and

$$
c(0, t)= \begin{cases}0 & \text { if } t<20 \\ 0.02(\cos (t / \pi))_{+} & \text {if } t \geq 20\end{cases}
$$

so that a wave of sediments is getting into the domain periodically.

The results are shown in Figures 12 to 16. Once more the E3W-HLLC and the 4W-HLLC solvers give practically the same results for the free surface and concentration (see Figs. 12 to 15) while some differences can be observed in the computed discharge (see Fig. 16): again 4W-HLLC is more accurate than E3W-HLLC.

While the CPU time required to solve this test with the Roe method is 166.55 , the corresponding to E3WHLLC and 4W-HLLC are 61.57 and 65.72 , respectively. This means roughly a $60 \%$ gain when using HLLC instead of Roe. Remark that E3W-HLLC is slightly faster as it does not need the computation of the intermediate velocity $S_{M}$. 


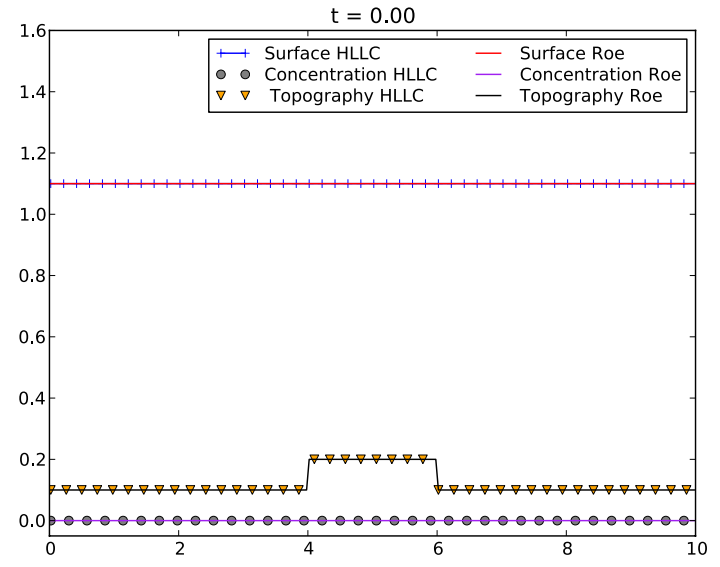

(a)

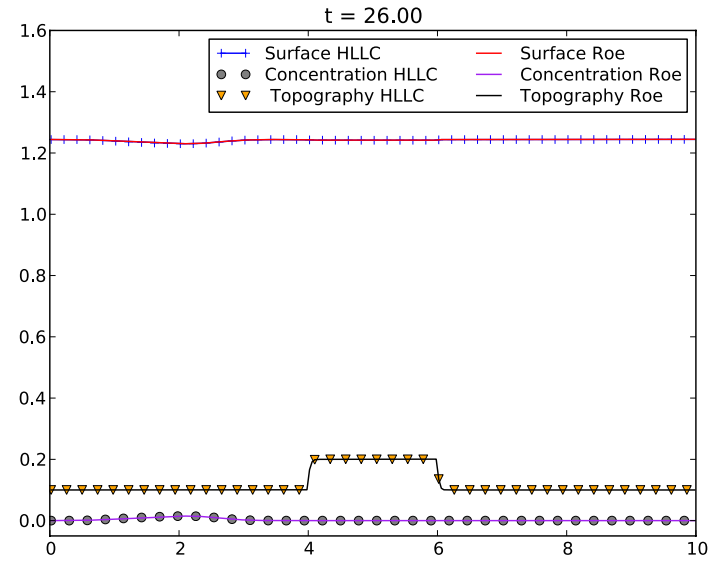

(b)

FiguRE 14. Moving bottom: 4W-HLLC.

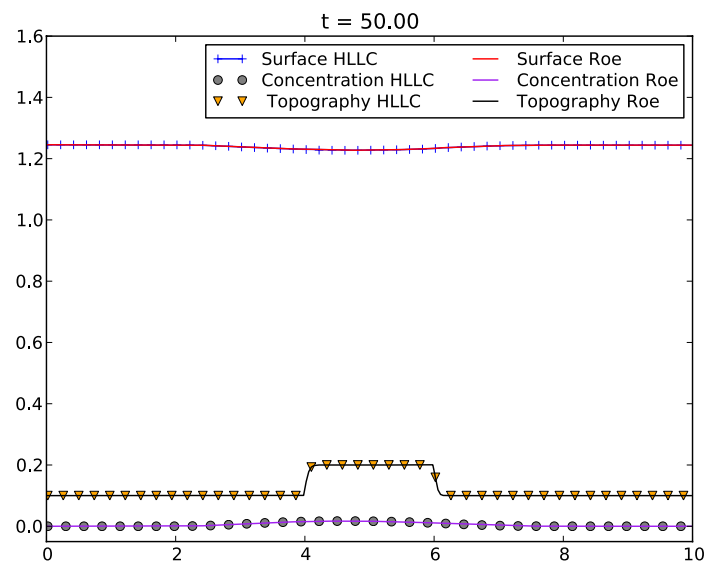

(a)

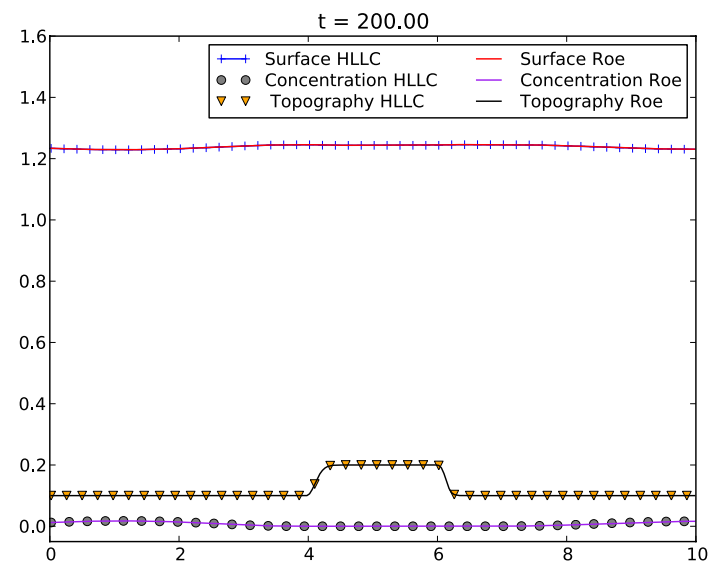

(b)

FiguRE 15. Moving bottom: 4W-HLLC.

\subsection{Hugoniot curves}

This test is designed to study the convergence error due to the numerical viscosity discussed in Section 1 . We consider $c=0$ and the Grass formula with $A=0.1$. Consider a given right state $h_{R}=4.8863763300885 e-1$, $u_{R}=-4.8295086427435 e-1,\left(z_{b}\right)_{R}=9.3440819151415 e-2$. Using the jump relations (3.17) we may compute the Hugoniot curve composed by the left states that can be connected to the fixed right state by an entropy shock associated to the characteristic field corresponding to the greatest eigenvalue (Lax's entropy conditions are considered here). In order to numerically compute the Hugoniot curve given by the numerical scheme, we solve a family of Riemann problems whose initial conditions are given by the fixed right state and some of the left states lying on the exact Hugoniot curve. In order to analyse the jump conditions satisfied by the limits of the numerical solutions for $4 \mathrm{~W}$-HLLC, the space step has been chosen small enough $(\Delta x=0.001)$ to ensure that the numerical solutions are close of the limit. At time $t=1$, the location of the shock is detected and the value of the left state is stored. In Figure 17 the theoretical and numerical Hugoniot curves are composed. 


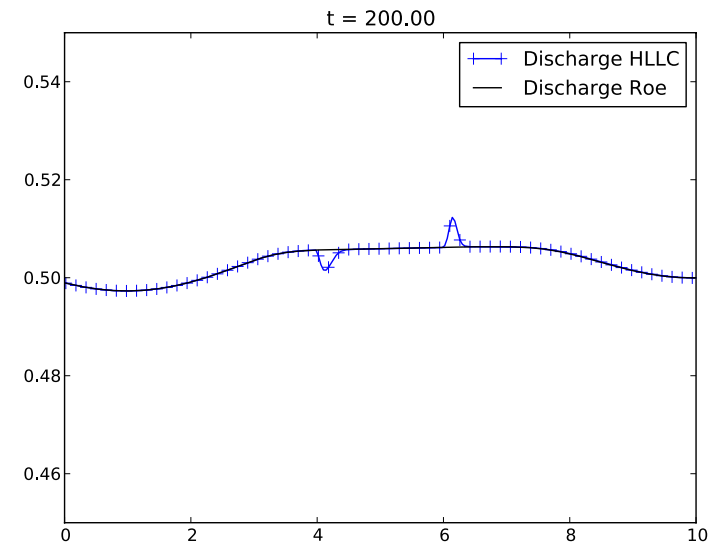

(a) E3W-HLLC

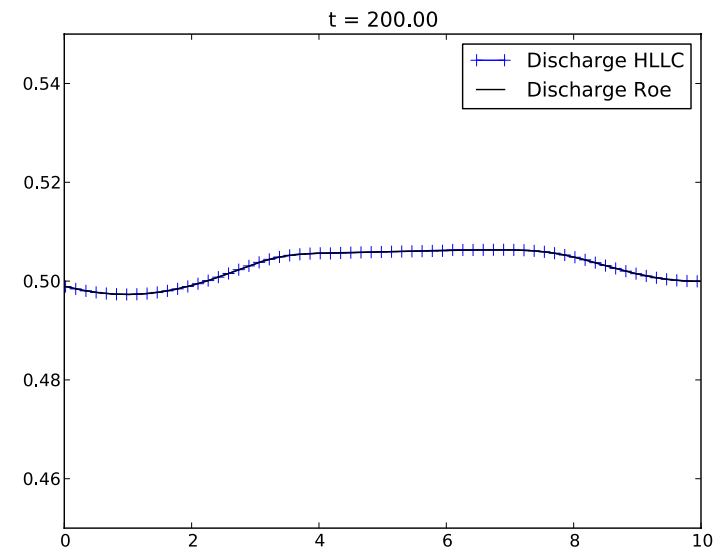

(b) $4 \mathrm{~W}$-HLLC

FiguRE 16. Moving bottom.

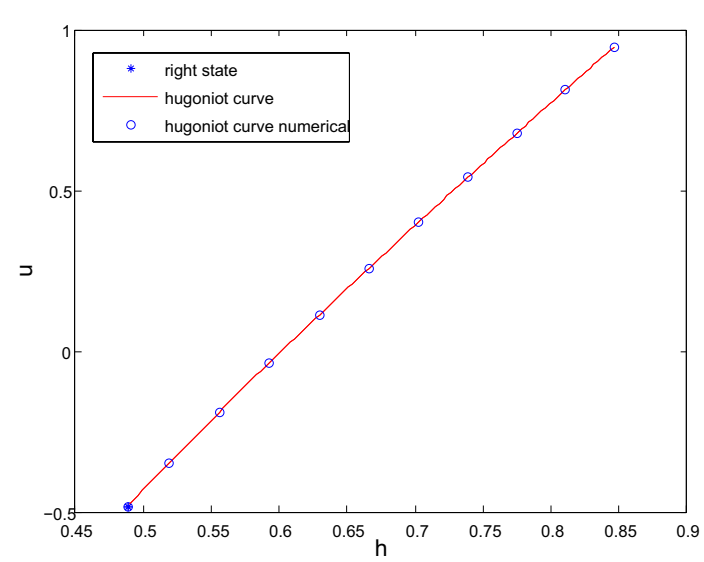

(a)

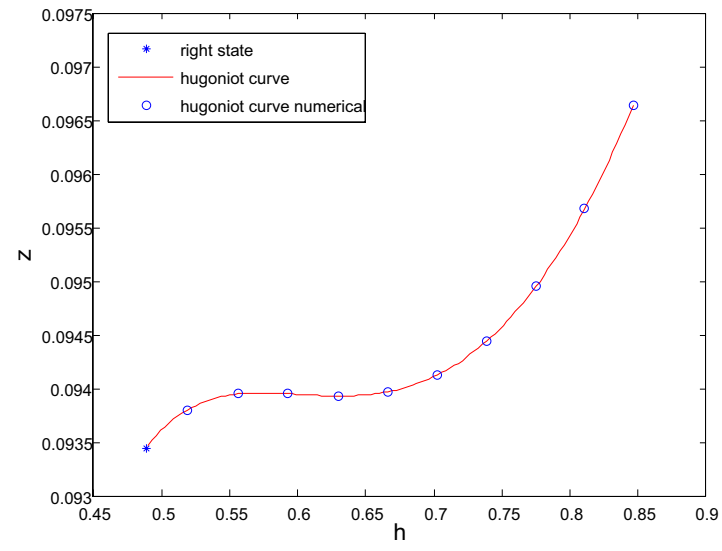

(b)

FiguRE 17. Hugoniot curves (3.17) for external shock.

Next a similar procedure has been applied to a Hugoniot curve corresponding to internal shocks, that is, entropy shocks associated to the characteristic field corresponding to the internal eigenvalue. In this case we fixed the left state $h_{L}=1, u_{L}=1, z_{L}=1$. Figure 18 shows the comparison at time $t=1$ of the right states obtained from (3.17) and computed numerically with 4 W-HLLC. In both cases a very good agreement is found.

\section{7. $2 \mathrm{~d}$ test case}

The scheme can be easily extended for the $2 \mathrm{~d}$ case and to higher order. We refer to $[6,12]$ for further details. Let us consider here a case of moving bottom. We consider the domain $D=[-1,1] \times[-1,1]$ and the initial condition

$$
\begin{gathered}
z_{b}(\vec{x}, 0)= \begin{cases}1.1+0.05 \cos (10|\vec{x}|) & \text { if }|\vec{x}| \leq \pi / 10, \\
1.05 & \text { otherwise. }\end{cases} \\
h(\vec{x}, 0)+z_{b}(\vec{x}, 0)=2, \quad q(\vec{x}, 0)=(1,0), \quad c(\vec{x}, 0)=0 ;
\end{gathered}
$$

for $\vec{x} \in[-1,1] \times[-1,1] \in \mathbb{R}^{2}$. Grass model will be considered with $A_{g}=0.01$. 


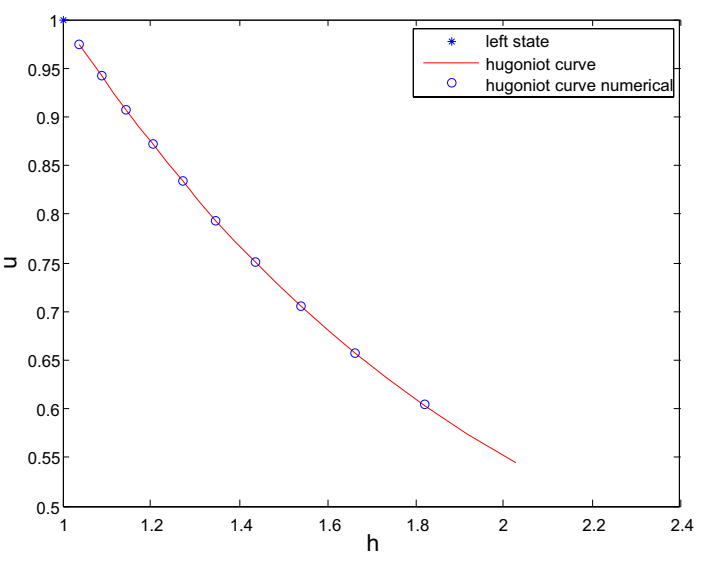

(a)

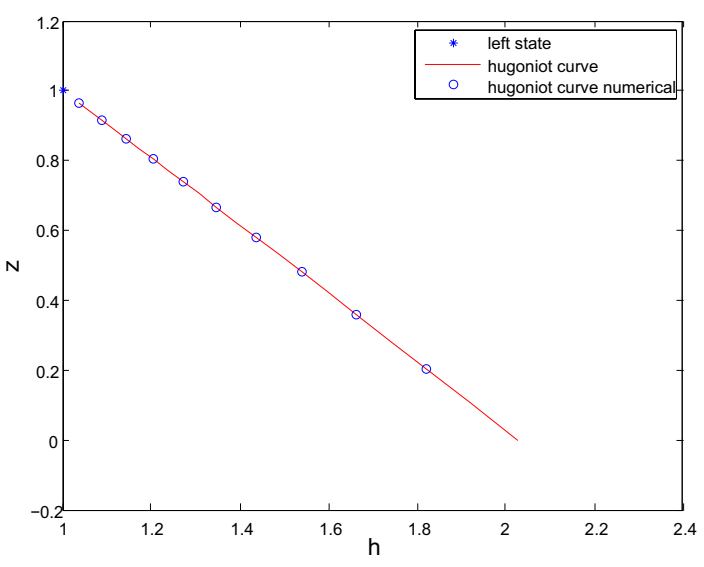

(b)

FigURE 18. Hugoniot curves (3.17) for internal shock.

TABLE 2. Comparison in $L^{1}$-norm between numerical solutions obtained with Roe and HLLC schemes at time $t=40 \mathrm{~s}$ for (5.11)-(5.13).

\begin{tabular}{|c|c|c|c|c|}
\hline & \multicolumn{2}{|c|}{ Test with (5.14) } & \multicolumn{2}{c|}{ Test with (5.15) } \\
\hline Variable & E3W-HLLC & 4 W-HLLC & E3W-HLLC & 4W-HLLC \\
\hline$h$ & $1.6802 \mathrm{e}-03$ & $6.4656 \mathrm{e}-05$ & $1.7768 \mathrm{e}-03$ & $1.0735 \mathrm{e}-04$ \\
\hline$q_{x}$ & $1.5328 \mathrm{e}-03$ & $3.2751 \mathrm{e}-05$ & $2.2334 \mathrm{e}-03$ & $6.2810 \mathrm{e}-05$ \\
\hline$q_{y}$ & $1.2679 \mathrm{e}-03$ & $2.6432 \mathrm{e}-05$ & $1.8904 \mathrm{e}-03$ & $4.1919 \mathrm{e}-05$ \\
\hline$h c$ & $3.3518 \mathrm{e}-05$ & $1.2921 \mathrm{e}-06$ & $4.7400 \mathrm{e}-05$ & $7.1250 \mathrm{e}-06$ \\
\hline$z_{b}$ & $8.0552 \mathrm{e}-04$ & $3.0761 \mathrm{e}-05$ & $1.7694 \mathrm{e}-03$ & $4.6355 \mathrm{e}-05$ \\
\hline
\end{tabular}

The following boundary conditions are imposed:

$$
\begin{gathered}
q_{y}(\vec{x}, t)=0, \quad \text { for } \vec{x} \in[-1,1] \times\{-1,1\}, t \geq 0, \\
q_{x}(\vec{x}, t)=1, \quad \text { for } \vec{x} \in\{-1\} \times[-1,1], t \geq 0,
\end{gathered}
$$

where $\vec{q}=\left(q_{x}, q_{y}\right)$. We shall consider two different test cases. In the first one, an homogeneous boundary condition on suspended sediment concentration is imposed. In the second one two different boundary conditions are considered for suspended sediment. The idea of the second test is to study the influence of different suspended sediment concentration on the evolution of the topography. More explicitly, in the first test we impose

$$
h c(\vec{x}, t)=0.019, \quad \text { for } \vec{x} \in\{-1\} \times[-1,1], t \geq 0,
$$

while for the second one, we impose the boundary condition

$$
\begin{gathered}
h c(\vec{x}, t)=0.04, \quad \text { for } \vec{x} \in\{-1\} \times[-1,0], t \geq 0, \\
h c(\vec{x}, t)=0.02, \quad \text { for } \vec{x} \in\{-1\} \times(0,1], t \geq 0 .
\end{gathered}
$$

Open boundary conditions are considered at the remaining boundaries.

We consider 100 points in the $x$ and $y$ direction respectively and a second order extension is used (see $[6,8,14]$ ).

The sediment layer evolves towards a star-shaped pattern expanding along the time. Figures 19 to 22 show topography, surface and discharge at time $t=40 \mathrm{~s}$ for the two different boundary conditions. 


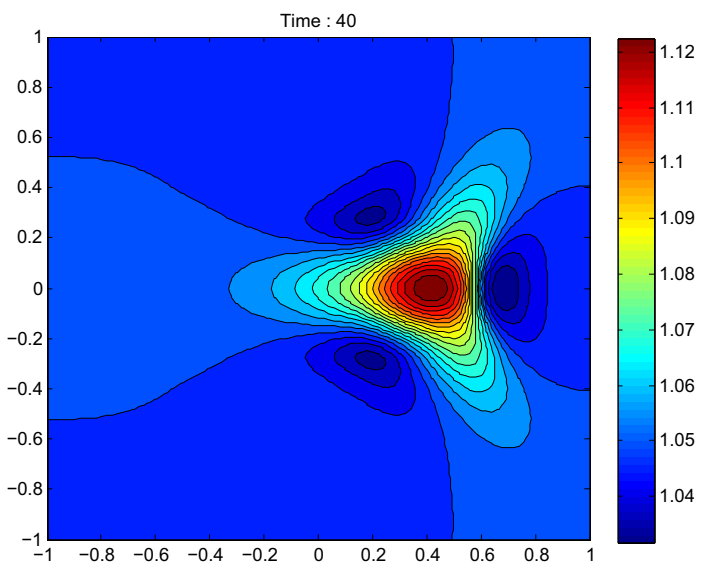

(a) Test with boundary condition (5.14)

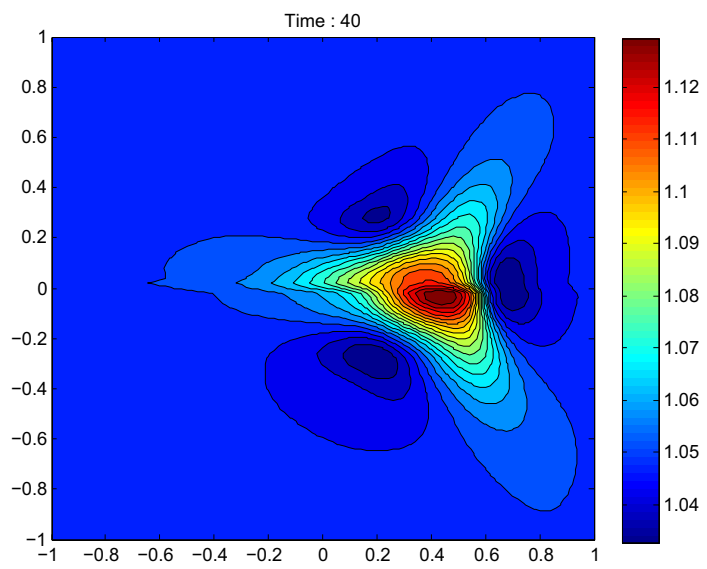

(b) Test with boundary condition (5.15)

Figure 19. Topography at time $t=40 \mathrm{~s}$ for (5.11)-(5.13).

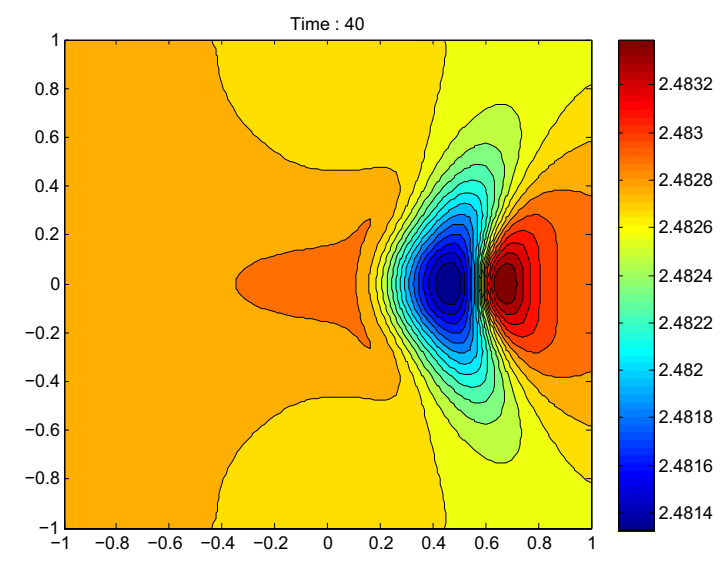

(a) Test with boundary condition (5.14)

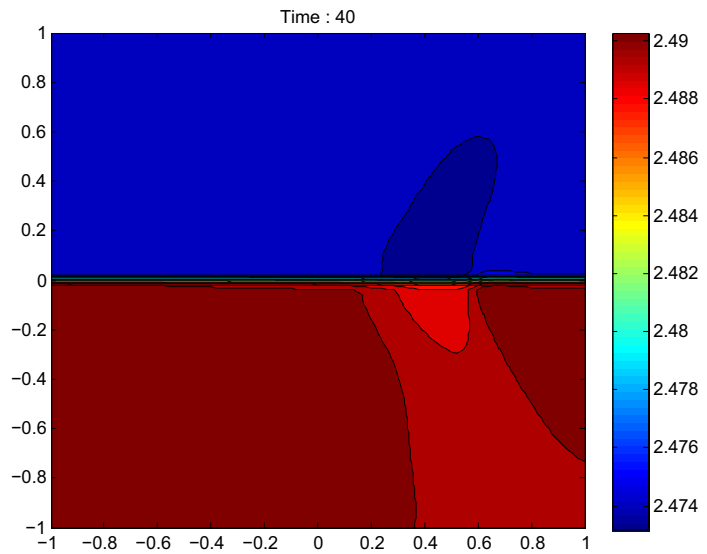

(b) Test with boundary condition (5.15)

Figure 20. Surface at time $t=40 \mathrm{~s}$ for (5.11)-(5.13).

If we compare the numerical solutions obtained for both HLLC schemes with the one given by Roe scheme, we get the $L^{1}$-norm errors given in Table 2 for both boundaries conditions.

We remark that numerical results obtained with both HLLC schemes introduced here are comparable to those obtained with Roe scheme and they are less expensive from the computational point of view. E3W-HLLC and $4 \mathrm{~W}$-HLLC need $63.37 \mathrm{~s}$ and $67.53 \mathrm{~s}$ of CPU time respectively while Roe scheme needs $157.26 \mathrm{~s}$. This means almost $60 \%$ gain when using HLLC instead of Roe. Nevertheless, the E3W-HLLC scheme is more diffusive than the other two ones, specially in the absence of the second order reconstruction technique.

\section{Conclusions}

In this paper two well-balanced HLLC solvers have been defined for the turbidity current model (2.8): the so-called essentially three-wave and the four-wave HLLC solvers. The first one preserves the non-negativity of 


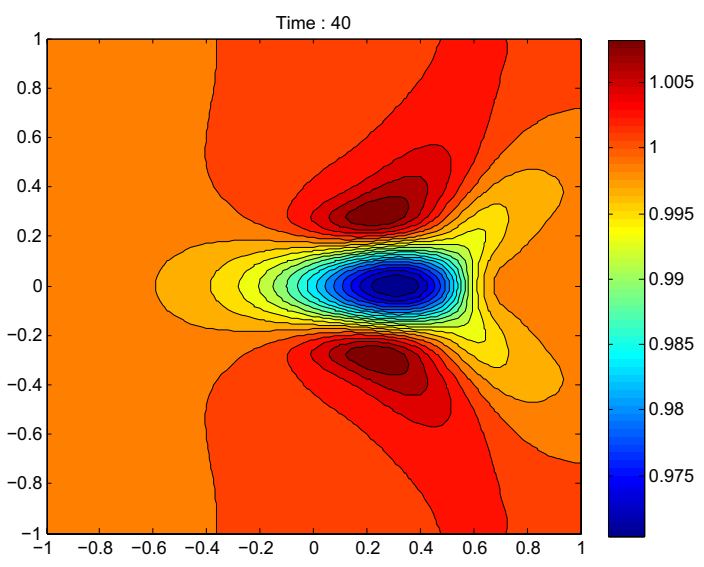

(a) Test with boundary condition (5.14)

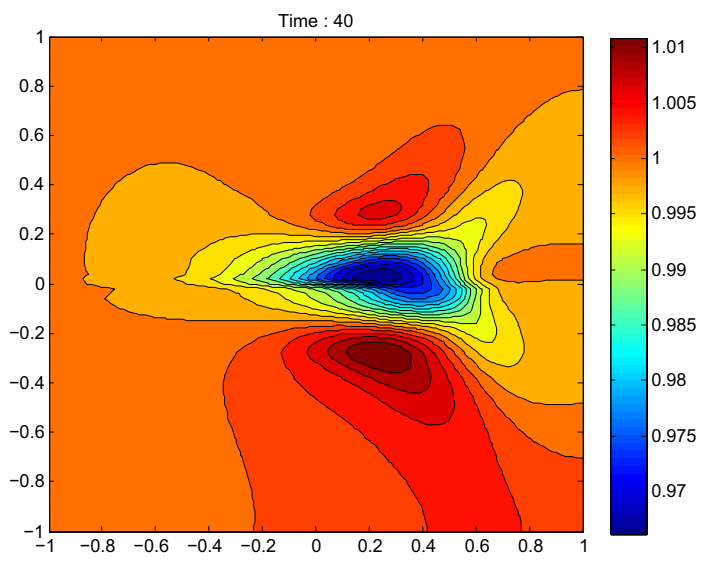

(b) Test with boundary condition (5.15)

Figure 21. Discharge on $x$ direction at time $t=40 \mathrm{~s}$ for $(5.11)-(5.13)$.

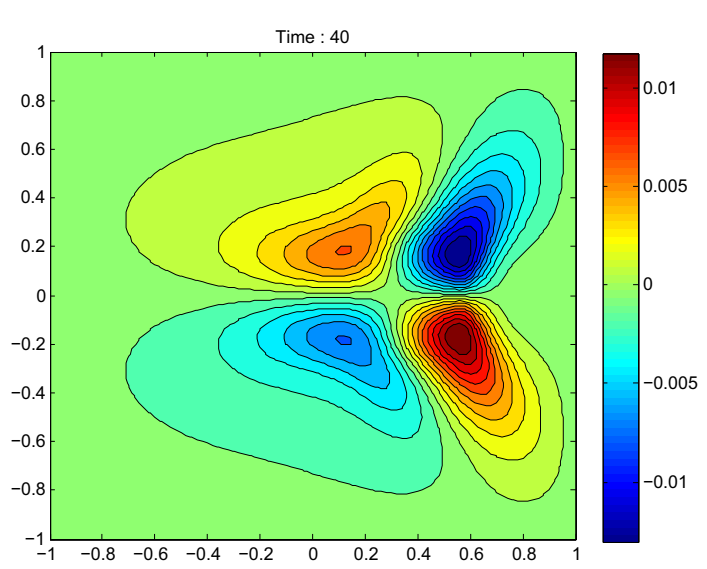

(a) Test with boundary condition (5.14)

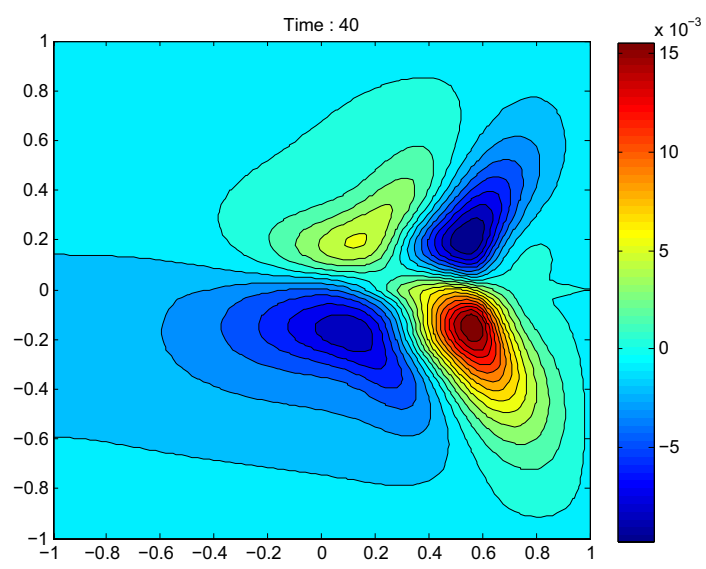

(b) Test with boundary condition (5.15)

FiguRE 22. Discharge on $y$ direction at time $t=40 \mathrm{~s}$ for (5.11)-(5.13).

the current thickness $h$. Under the assumption that the solid discharge formula $q_{b}$ only depends on the velocity of the turbidity current, the non-negativity of the thickness of the sediment layer is also ensured. Moreover, for a fixed flat topography it is entropy satisfying as in that case it coincides with the Suliciu relaxation scheme introduced in [3]. Some conditions that implies the non-negativity of $h$ are also derived for the four-wave HLLC solver if $q_{b}=0$, but these conditions may not be satisfied in practice in the presence of large steps in the bottom. Although the numerical results provided by both solvers are close, the four-wave solver is slightly more accurate and more costly. Moreover, the results are also close to those provided by the Roe scheme introduced in [23], but the computational cost is much lower, as the HLLC solvers do not need the explicit knowledge of the complete eigenstructure of the system: a speedup of about $60 \%$ is obtained for the HLLC solvers compared to Roe method. 


\section{Appendix A. Relation Between different Jump COnditions}

Any jump conditions related to a particular choice of paths is expected to give a third order approximation of the physically correct ones in the following sense:

Theorem A.1. Let us suppose that $A: \Omega \mapsto \mathbb{R}^{N \times N}$ has continuous second order derivatives. Let $W_{L}$ and $W_{R}$ be a pair of states of $\Omega$ such that there exists $\lambda$ satisfying

$$
\left.\lambda\left(W_{R}-W_{L}\right)=\int_{0}^{1} A\left(\Psi\left(s ; W_{L}, W_{R}\right)\right) \Psi_{s}\left(s ; W_{L}, W_{R}\right)\right) \mathrm{d} s .
$$

Given any other family of paths $\Phi:[0,1] \times \Omega \times \Omega \mapsto \Omega$ with continuous second order derivatives, one has:

$$
\left.\lambda\left(W_{R}-W_{L}\right)=\int_{0}^{1} A\left(\Phi\left(s ; W_{L}, W_{R}\right)\right) \Phi_{s}\left(s ; W_{L}, W_{R}\right)\right) \mathrm{d} s+O\left(\left|W_{R}-W_{L}\right|^{3}\right) .
$$

Proof. Let us denote by:

$$
\gamma \in[0, M] \mapsto \phi(\gamma)
$$

the arc-length parametrization of the path:

$$
s \in[0,1] \mapsto \Phi\left(s ; W_{L}, W_{R}\right),
$$

whose length is denoted by $M$. By applying the midpoint rule and some Taylor expansions we obtain:

$$
\begin{aligned}
\left.\int_{0}^{1} A\left(\Phi\left(s ; W_{L}, W_{R}\right)\right) \Phi_{s}\left(s ; W_{L}, W_{R}\right)\right) & =\int_{0}^{M} A(\phi(\gamma)) \phi^{\prime}(\gamma) \mathrm{d} \gamma \\
& =M A\left(\phi\left(\frac{M}{2}\right)\right) \phi^{\prime}\left(\frac{M}{2}\right)+O\left(M^{3}\right) \\
& =A\left(\phi\left(\frac{M}{2}\right)\right)(\phi(M)-\phi(0))+O\left(M^{3}\right) \\
& =A\left(\phi\left(\frac{M}{2}\right)\right)\left(W_{R}-W_{L}\right)+O\left(M^{3}\right) \\
& =A\left(\frac{1}{2}(\phi(0)+\phi(M))\right)\left(W_{R}-W_{L}\right)+\left(W_{R}-W_{L}\right) O\left(M^{2}\right)+O\left(M^{3}\right) \\
& =A\left(\frac{1}{2}\left(W_{L}+W_{R}\right)\right)\left(W_{R}-W_{L}\right)+\left(W_{R}-W_{L}\right) O\left(M^{2}\right)+O\left(M^{3}\right) .
\end{aligned}
$$

From the definition of a family of paths (see [10]), there exists a constant $K$ such that:

$$
\left|\partial_{s} \Phi\left(s ; W_{L}, W_{R}\right)\right| \leq K\left|W_{R}-W_{L}\right|, \quad \forall s .
$$

By integrating in $[0,1]$ we obtain:

$$
M \leq K\left|W_{R}-W_{L}\right| .
$$

Then, the following equality can be deduced:

$$
\int_{0}^{1} A\left(\Phi\left(s ; W_{L}, W_{R}\right)\right) \Phi_{s}\left(s ; W_{L}, W_{R}\right)=A\left(\frac{1}{2}\left(W_{L}+W_{R}\right)\right)\left(W_{R}-W_{L}\right)+O\left(\left|W_{R}-W_{L}\right|^{3}\right)
$$

for every smooth enough family of segments. In particular, it holds for $\Psi$ :

$$
\int_{0}^{1} A\left(\Psi\left(s ; W_{L}, W_{R}\right)\right) \Psi_{s}\left(s ; W_{L}, W_{R}\right)=A\left(\frac{1}{2}\left(W_{L}+W_{R}\right)\right)\left(W_{R}-W_{L}\right)+O\left(\left|W_{R}-W_{L}\right|^{3}\right) .
$$


The proof is then finished from (A.1) and (A.2):

$$
\begin{aligned}
\lambda\left(W_{R}-W_{L}\right) & \left.=\int_{0}^{1} A\left(\Psi\left(s ; W_{L}, W_{R}\right)\right) \Psi_{s}\left(s ; W_{L}, W_{R}\right)\right) \\
& =A\left(\frac{1}{2}\left(W_{L}+W_{R}\right)\right)\left(W_{R}-W_{L}\right)+O\left(\left|W_{R}-W_{L}\right|^{3}\right) \\
& \left.=\int_{0}^{1} A\left(\Phi\left(s ; W_{L}, W_{R}\right)\right) \Phi_{s}\left(s ; W_{L}, W_{R}\right)\right)+O\left(\left|W_{R}-W_{L}\right|^{3}\right) .
\end{aligned}
$$

Remark A.2. Notice that a family of paths $\Phi$ based on the viscous profiles or a regularized system is expected to be smooth.

\section{Appendix B. Proof of Theorem 4.3}

For the sake of completeness we give here the proof. First, let us remark that $p(h, c)$ defined by $(4.9)$ verifies that

$$
\begin{gathered}
\forall h, c>0, \quad \frac{\partial}{\partial h}\left(h \sqrt{\frac{\partial p}{\partial h}(h, c)}\right)>0, \\
h \sqrt{\frac{\partial p}{\partial h}(h, c)} \rightarrow \infty \text { as } h \rightarrow \infty, \\
\frac{\partial}{\partial h}\left(h \sqrt{\frac{\partial p}{\partial h}(h, c)}\right) \leq \alpha \sqrt{\frac{\partial p}{\partial h}(h, c),}
\end{gathered}
$$

where $\alpha=3 / 2$.

(B.1)-(B.2) allow us to define an inverse function $\psi(\cdot, c):(0, \infty) \rightarrow(0, \infty)$ for each $c$,

$$
h \sqrt{\frac{\partial p}{\partial h}(h, c)}=k \Leftrightarrow h=\psi(h, k)
$$

Then, we have the following lemma:

Lemma B.1. Assume $k_{R}>0$ and define

$$
\frac{k_{L}}{h_{L}}=\sqrt{\frac{\partial p}{\partial h}\left(h_{L}, c_{L}\right)}+\alpha\left(\frac{\mathcal{P}_{L R}}{k_{R}}+u_{L}-u_{R}\right)_{+},
$$

then

$$
\frac{1}{h_{L}}+\frac{k_{R}\left(u_{R}-u_{L}\right)-\mathcal{P}_{L R}}{k_{L}\left(k_{L}+k_{R}\right)} \geq \frac{1}{\psi\left(k_{L}, c_{L}\right)}
$$

Proof. Suppose first that

$$
k_{R}\left(u_{R}-u_{L}\right)-\mathcal{P}_{L R} \geq 0
$$

then

$$
k_{L}=h_{L} \sqrt{\frac{\partial p}{\partial h}\left(h_{L}, c_{L}\right)} \Leftrightarrow \psi\left(k_{L}, c_{L}\right)=h_{L}
$$

and the result follows. Assume now

$$
k_{R}\left(u_{R}-u_{L}\right)-\mathcal{P}_{L R}<0,
$$


and define

$$
X=\frac{\mathcal{P}_{L R}}{k_{R}}+u_{L}-u_{R}>0 .
$$

Then, (B.6) is equivalent to

$$
1-\frac{k_{R}}{k_{L}+k_{R}} \frac{X}{\sqrt{\frac{\partial p}{\partial h}\left(h_{L}, c_{L}\right)}+\alpha X} \geq \frac{h_{L}}{\psi\left(k_{L}, c_{L}\right)} .
$$

Denote by

$$
\theta=\frac{\sqrt{\frac{\partial p}{\partial h}\left(h_{L}, c_{L}\right)}}{\sqrt{\frac{\partial p}{\partial h}\left(h_{L}, c_{L}\right)}+\alpha X}, \quad 1-\theta=\frac{\alpha X}{\sqrt{\frac{\partial p}{\partial h}\left(h_{L}, c_{L}\right)}+\alpha X}
$$

It is enough to prove

$$
1-\frac{1-\theta}{\alpha}-\frac{h_{L}}{\psi\left(h_{L}\left(\sqrt{\frac{\partial p}{\partial h}\left(h_{L}, s_{L}\right)}+\alpha X\right), c_{L}\right)} \geq 0 .
$$

From (B.3) we get

$$
\frac{\partial \psi}{\partial k}(k, c) \geq \frac{\psi(k, c)}{\alpha k}
$$

and

$$
\frac{\partial}{\partial k}\left(\psi(k, c) k^{-1 / \alpha}\right) \geq 0
$$

so that

$$
\forall \lambda \geq 1, \quad \psi(\lambda k, c) \geq \lambda^{1 / \alpha} \psi(k, c)
$$

Thus, it is enough to prove

$$
1-\frac{1-\theta}{\alpha}-\theta^{1 / \alpha} \geq 0
$$

and this is indeed the case when $0<\theta \leq 1$ and $\alpha \geq 1$.

Remark B.2. The result of the lemma can be symmetrized and for any $k_{L}>0$, the value

$$
\frac{k_{R}}{h_{R}}=\sqrt{\frac{\partial p}{\partial h}\left(h_{R}, c_{R}\right)}+\alpha\left(-\frac{\mathcal{P}_{L R}}{k_{L}}+u_{L}-u_{R}\right)_{+},
$$

satisfies

$$
\frac{1}{h_{R}}+\frac{k_{L}\left(u_{R}-u_{L}\right)+\mathcal{P}_{L R}}{k_{R}\left(k_{L}+k_{R}\right)} \geq \frac{1}{\psi\left(k_{R}, c_{R}\right)}
$$

Proof of Theorem 4.3. Let us assume that $\mathcal{P}_{L R}>0$, the other case being analogous. By Remark B.2 we have

$$
\frac{1}{h_{R}}+\frac{k_{L}\left(u_{R}-u_{L}\right)+\mathcal{P}_{L R}}{k_{R}\left(k_{L}+k_{R}\right)} \geq \frac{1}{\psi\left(k_{R}, c_{R}\right)}
$$

Now, if $k_{R}\left(u_{R}-u_{L}\right)-\mathcal{P}_{L R} \geq 0$, then

$$
\frac{1}{h_{L}}+\frac{k_{R}\left(u_{R}-u_{L}\right)-\mathcal{P}_{L R}}{k_{L}\left(k_{L}+k_{R}\right)} \geq \frac{1}{h_{L}} \geq \frac{1}{\psi\left(k_{L}, c_{L}\right)} .
$$


If $k_{R}\left(u_{R}-u_{L}\right)-\mathcal{P}_{L R}<0$, then

$$
k_{R} \geq h_{R} \sqrt{\frac{\partial p}{\partial h}\left(h_{R}, c_{R}\right)} \text { and } \mathcal{P}_{L R} \geq 0
$$

and we have $k_{L} \geq \widehat{k}_{L}$ with

$$
\frac{\widehat{k}_{L}}{h_{L}}=\sqrt{\frac{\partial p}{\partial h}\left(h_{L}, c_{L}\right)}+\alpha\left(\frac{\mathcal{P}_{L R}}{k_{R}}+u_{L}-u_{R}\right)_{+} .
$$

By applying Lemma B.1 to the pair $\left(\widehat{k}_{L}, k_{R}\right)$ we have

$$
\frac{1}{h_{L}}+\frac{k_{R}\left(u_{R}-u_{L}\right)-\mathcal{P}_{L R}}{\widehat{k}_{L}\left(\widehat{k}_{L}+k_{R}\right)} \geq \frac{1}{\psi\left(\widehat{k}_{L}, c_{L}\right)},
$$

and by using the fact that $k_{R}\left(u_{R}-u_{L}\right)-\mathcal{P}_{L R}<0$ and that $f(k)=-1 /\left(k\left(k+k_{R}\right)\right)$ is an increasing function of $k \in(0, \infty)$ we finally obtain

$$
\frac{1}{h_{L}}+\frac{k_{R}\left(u_{R}-u_{L}\right)-\mathcal{P}_{L R}}{k_{L}\left(k_{L}+k_{R}\right)} \geq \frac{1}{h_{L}}+\frac{k_{R}\left(u_{R}-u_{L}\right)-\mathcal{P}_{L R}}{\widehat{k}_{L}\left(\widehat{k}_{L}+k_{R}\right)} \geq \frac{1}{\psi\left(\widehat{k}_{L}, c_{L}\right)} \geq \frac{1}{\psi\left(k_{L}, c_{L}\right)} .
$$

Remark B.3. We have actually proved that

$$
\frac{1}{h_{L}}+\frac{k_{R}\left(u_{R}-u_{L}\right)-\mathcal{P}_{L R}}{k_{L}\left(k_{L}+k_{R}\right)} \geq \frac{1}{\psi\left(k_{L}, c_{L}\right)}, \frac{1}{h_{R}}+\frac{k_{L}\left(u_{R}-u_{L}\right)+\mathcal{P}_{L R}}{k_{R}\left(k_{L}+k_{R}\right)} \geq \frac{1}{\psi\left(k_{R}, c_{R}\right)} .
$$

which can be used to show that the numerical scheme for fixed flat topography is entropy satisfying.

\section{Appendix C. Proof of Proposition 4.4}

Let us first remark that

$$
\tilde{q}_{b}^{*}=\phi\left(u_{L}^{*}\right)=\phi\left(u_{R}^{*}\right)=\tilde{q}_{b}^{*},
$$

what gives $z_{b}^{*}=z_{b}^{*}$. Next, (4.8) can be rewritten as follows:

$$
z_{b L}^{M}=\frac{1-\frac{\tilde{q}_{b}}{u_{L}-k_{L} / h_{L}}}{1-\frac{\phi\left(u_{L}^{*}\right)}{u_{L}-k_{L} / h_{L}}} z_{b L} .
$$

Since $\tilde{q}_{b}$ is bounded, we have:

$$
\frac{\tilde{q}_{b}}{u_{L}-k_{L} / h_{L}}<1
$$

if $k_{L}$ is large enough. On the other hand, as

$$
u_{L}^{*}=\frac{k_{L}}{k_{L}+k_{R}} u_{L}+\frac{k_{R}}{k_{L}+k_{R}} u_{R}-\frac{\mathcal{P}_{L R}}{k_{L}+k_{R}}
$$

and $\mathcal{P}_{L R}$ does not depend on $k_{L}$ and $k_{R}$, we deduce that $u_{L}^{*}$ and $\phi\left(u_{L}^{*}\right)$ remain bounded for large values of $k_{L}$ and $k_{R}$. Therefore, we can choose these values large enough so that:

$$
\frac{\phi\left(u_{L}^{*}\right)}{u_{L}-k_{L} / h_{L}}<1
$$

and thus $z_{b}^{M} \geq 0$. A similar argument shows that $z_{b}{ }_{R}^{*} \geq 0$. 


\section{Appendix D. Proof of Proposition 4.7}

The following equalities can be shown:

$$
h_{L}^{*}=\frac{h_{L}\left(u_{L}-S_{L}\right)+S_{L} h_{L}^{M}}{S^{*}}, \quad h_{R}^{*}=\frac{h_{R}\left(u_{R}-S_{R}\right)}{S^{*}-S_{R}} .
$$

The non-negativity of $h_{L}^{*}$ is then deduced from the upper bound in (4.25) and $h_{R}^{*}$ is non-negative as $u_{R}-S_{R} \leq 0$ and $S^{*} \leq S_{R}$.

If $S_{L}$ and $S_{R}$ are written as $S_{L}=u_{L}-\frac{k_{L}}{h_{L}}$ and $S_{R}=u_{R}+\frac{k_{R}}{h_{R}}$, as it was done in Theorem 4.3, one has:

$$
\begin{aligned}
S^{*}-S_{R} & =\frac{h_{R} u_{R}\left(S_{R}-u_{R}\right)+h_{L}\left(u_{L}^{2}-S_{L}^{2}\right)+S_{L}^{2} h_{L}^{M}-\mathcal{P}_{L R}-S_{R}\left(h_{R}\left(S_{R}-u_{R}\right)+h_{L}\left(u_{L}-S_{L}\right)+S_{L} h_{L}^{M}\right)}{h_{R}\left(S_{R}-u_{R}\right)+h_{L}\left(u_{L}-S_{L}\right)+S_{L} h_{L}^{M}} \\
& =\frac{h_{R}\left(u_{R} S_{R}-u_{R}^{2}-S_{R}^{2}+S_{R} u_{R}\right)+h_{L}\left(u_{L}^{2}-S_{L}^{2}-S_{R} u_{L}+S_{R} S_{L}\right)+S_{L}\left(S_{L}-S_{R}\right) h_{L}^{M}-\mathcal{P}_{L R}}{h_{R}\left(S_{R}-u_{R}\right)+h_{L}\left(u_{L}-S_{L}\right)+S_{L} h_{L}^{M}} .
\end{aligned}
$$

Now remark that

$$
\frac{h_{R}\left(S_{R}-u_{R}\right)+h_{L}\left(u_{L}-S_{L}\right)}{-S_{L}} \geq \frac{h_{L}\left(u_{L}-S_{L}\right)}{-S_{L}} \geq h_{L}^{M},
$$

what implies that $S^{*}-S_{R} \leq 0$ as long as the numerator in the right hand side of (D.1) is non positive.

Some easy calculations show that this is the case when

$$
h_{L}^{M} \leq \frac{h_{R}\left(u_{R}-S_{R}\right)^{2}+h_{L}\left(u_{L}-S_{L}\right)^{2}+h_{L} u_{L}\left(S_{R}+2 S_{L}-2 u_{L}\right)-h_{L} S_{L} S_{R}-\mathcal{P}_{L R}}{-S_{L}\left(S_{R}-S_{L}\right)}
$$

what completes the proof.

Acknowledgements. This research has been partially supported by the Spanish Government and FEDER through the Research projects MTM2009-11923, MTM2009-07719, and by the Andalusian Government through the project P06RNM-01594. The numerical computations have been performed at the Laboratory of Numerical Methods of the University of Málaga.

\section{REFERENCES}

[1] R. Abgrall and S. Karni, A comment on the computation of non-conservative products. J. Comput. Phys. 229 (2010) 2759-2763.

[2] M.S. Altinaker, W.H. Graf and E. Hopfinger, Flow structure in turbidity currents. J. Hydr. Res. 34 (1996) $713-718$.

[3] F. Bouchut, Nonlinear stability of finite volume methods for hyperbolic conservation laws and well-balanced schemes for sources, in Frontiers in Mathematics. Birkhäuser Verlag, Basel (2004).

[4] S.F. Bradford and N.D. Katopodes, Hydrodynamics of turbid underflows. i: Formulation and numerical analysis. J. Hydr. Eng. 125 (1999) 1006-1015.

[5] M.J. Castro, P.G. LeFloch, M.L. Muñoz-Ruiz and C. Parés, Why many theories of shock waves are necessary: Convergence error in formally path-consistent schemes. J. Comput. Phys. 227 (2008) 8107-8129.

[6] M.J. Castro, E.D. Fernández-Nieto, A.M. Ferreiro, J.A. García-Rodríguez and C. Parés, High order extensions of Roe schemes for two-dimensional nonconservative hyperbolic systems. J. Sci. Comput. 39 (2009) 67-114.

[7] M. Castro Díaz, E. Fernéndez-Nieto and A. Ferreiro, Sediment transport models in shallow water equations and numerical approach by high order finite volume methods. Comput. Fluids 37 (2008) 299-316.

[8] M.J. Castro Díaz, E.D. Fernández-Nieto, A.M. Ferreiro and C. Parés, Two-dimensional sediment transport models in shallow water equations. A second order finite volume approach on unstructured meshes. Comput. Methods Appl. Mech. Eng. 198 (2009) 2520-2538. 
[9] S. Cordier, M. Le and T. Morales de Luna, Bedload transport in shallow water models: Why splitting (may) fail, how hyperbolicity (can) help. Adv. Water Resour. 34 (2011) 980-989.

[10] G. Dal Maso, P.G. Lefloch and F. Murat, Definition and weak stability of nonconservative products. J. Math. Pures Appl. 74 (1995) 483-548.

[11] F. Exner, Über die wechselwirkung zwischen wasser und geschiebe in flüssen. Sitzungsber., Akad. Wissenschaften IIa (1925).

[12] E.D. Fernández-Nieto, Modelling and numerical simulation of submarine sediment shallow flows: transport and avalanches. Bol. Soc. Esp. Mat. Apl. SëMA 49 (2009) 83-103.

[13] A.C. Fowler, N. Kopteva and C. Oakley, The formation of river channels. SIAM J. Appl. Math. 67 (2007) 1016-1040.

[14] J. Gallardo, S. Ortega, M. de la Asunción and J. Mantas, Two-Dimensional compact third-order polynomial reconstructions. solving nonconservative hyperbolic systems using GPUs. J. Sci. Comput. 48 (2011) 141-163.

[15] A. Grass, Sediment transport by waves and currents. SERC London Cent. Mar. Technol. Report No. FL29 (1981).

[16] A. Harten, P.D. Lax and B. van Leer, On upstream differencing and godunov-type schemes for hyperbolic conservation laws. SIAM Rev. 25 (1983).

[17] T.Y. Hou and P.G. Le Floch, Why nonconservative schemes converge to wrong solutions: error analysis. Math. Comput. 62 (1994) 497-530.

[18] S.M. Khan, J. Imran, S. Bradford and J. Syvitski, Numerical modeling of hyperpycnal plume. Mar. Geol. 222-223 (2005) 193-211.

[19] Y. Kubo, Experimental and numerical study of topographic effects on deposition from two-dimensional, particle-driven density currents. Sediment. Geol. 164 (2004) 311-326.

[20] Y. Kubo and T. Nakajima, Laboratory experiments and numerical simulation of sediment-wave formation by turbidity currents. Mar. Geol. 192 (2002) 105-121.

[21] D.A. Lyn and M. Altinakar, St. Venant-Exner equations for Near-Critical and transcritical flows. J. Hydr. Eng. 128 (2002) $579-587$.

[22] E. Meyer-Peter and R. Müller, Formulas for bed-load transport, in 2nd meeting IAHSR. Stockholm, Sweden (1948) 1-26.

[23] T. Morales de Luna, M.J. Castro Díaz, C. Parés Madroñal and E.D. Fernández Nieto, On a shallow water model for the simulation of turbidity currents. Commun. Comput. Phys. 6 (2009) 848-882.

[24] T. Morales de Luna, M.J. Castro Díaz and C. Parés Madroñal, A duality method for sediment transport based on a modified Meyer-Peter \& Müller model. J. Sci. Comput. 48 (2010) 258-273.

[25] P.H. Morris and D.J. Williams, Relative celerities of mobile bed flows with finite solids concentrations. J. Hydr. Eng. 122 (1996) 311-315.

[26] M.L. Muñoz Ruiz and C. Parés, On the convergence and Well-Balanced property of Path-conservative numerical schemes for systems of balance laws. J. Sci. Comput. 48 (2011) 274-295.

[27] P. Nielsen, Coastal Bottom Boundary Layers and Sediment Transport. World Scientific Pub. Co. Inc. (1992).

[28] C. Parés, Numerical methods for nonconservative hyperbolic systems: a theoretical framework. SIAM J. Numer. Anal. 44 (2006) 300-321 (electronic).

[29] C. Parés and M.L. Muñoz Ruiz, On some difficulties of the numerical approximation of nonconservative hyperbolic systems. Bol. Soc. Esp. Mat. Apl. 47 (2009) 23-52.

[30] G. Parker, Y. Fukushima and H.M. Pantin, Self-accelerating turbidity currents. J. Fluid Mech. 171 (1986) $145-181$.

[31] E.F. Toro, M. Spruce and W. Speares, Restoration of the contact surface in the HLL-Riemann solver. Shock Waves 4 (1994) 25-34.

[32] E.F. Toro, Shock-capturing methods for free-surface shallow flows. John Wiley (2001).

[33] L. Van Rijn, Sediment transport: bed load transport. J. Hydr. Eng. 110 (1984) 1431-1456. 